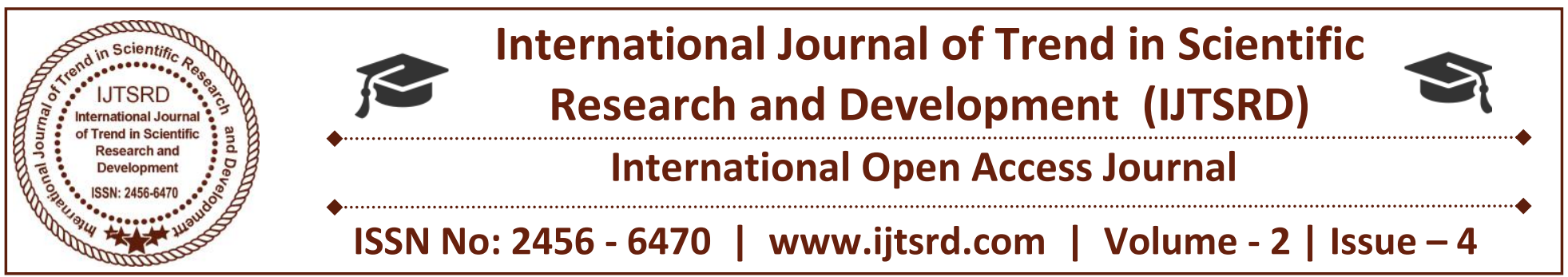

\title{
Protineuria in CKD Patient on Hemodialysis
}

\author{
Hulsi Sahu ${ }^{1}$, Atul Verma ${ }^{1}$, Hemin Sahu ${ }^{2}$ \\ ${ }^{1}$ Dialysis Technologist, ${ }^{2}$ Physiotherapist \\ ${ }^{1}$ Pt. J. N. M. Medical College, Raipur, Chhattisgarh, India \\ ${ }^{2}$ Shree Narayana Hospital, Raipur, Chhattisgarh, India
}

\begin{abstract}
Proteinuria is a distinguishing feature in primary and secondary forms of chronic glomerulonephritis, which contribute to no more than the $20 \%$ of the end-stage kidney disease (ESKD) population. The contribution of non-proteinuric nephropathies to the global ESKD burden is still poorly focused and scarce research efforts are dedicated to the elucidation of risk factors and mechanistic pathways triggering ESKD in these diseases. We abstracted information on proteinuria in the main renal diseases other than glomerulonephritides that may evolve into ESKD. In type 2 diabetes, non-proteinuric diabetic kidney disease (DKD) is more frequent than proteinuric $\mathrm{DKD}$, and risk factors for non-proteinuric forms of DKD now receive increasing attention. Similarly, proteinuria is most often inconspicuous or absent in the most frequent cause of ESKD, i.e. hypertensionrelated chronic kidney disease (CKD), as well as in progressive cystic diseases like autosomal dominant polycystic kidney disease and in pyelonephritis/ tubulo-interstitial diseases. Maintaining a high degree of attention in the care of CKD patients with proteinuria is fundamental to effectively retard progression toward kidney failure. However, substantial research efforts are still needed to develop treatment strategies that may help the vast majority of CKD patients who eventually develop ESKD via mechanistic pathways other than proteinuria.
\end{abstract}

Keywords: CKD, ESKD, non-proteinuric diseases, proteinuria

\section{INTRODUCTION}

\section{GENERAL INTRODUCTION}

In end stage kidney disease (ESKD) patients, mortality due to cardiovascular disease (CVD) is 10 to
30 times higher than in the general population. For example, a 30-year-old dialysis patient has a similar CVD mortality risk to that of an 80-year-old in the general population. This increased risk likely reflects the very high prevalence of CVD, increased prevalence and severity of diabetes, hypertension and left ventricular hypertrophy, and non-traditional risk factors such as chronic volume overload, hyperphosphatemia, anaemia, oxidant stress, and other aspects of the uremic milieu. In this chapter, we focus on epidemiology and management of traditional and non-traditional CVD risk factors, and on ischemic heart disease, heart failure, pericardial effusion, valvular disease, and arrhythmia. T. Daugridas peter.

Chronic kidney disease (CKD) is a worldwide health problem, affecting millions of people (Di Angelantonio et al., 2007). The magnitude of the problem is poorly described by the number of people that will initiate renal replacement therapy (haemodialysis, peritoneal dialysis and renal transplantation), as the incidence of $1-3$ per 10,000 per year in the general population may seem small). However, chronic dialysis treatment and transplantation have an enormous impact on the life of individual patients and their families, and renal replacement therapy is very costly (Lysaght, 2002; Dor et al., 2007). The annual worldwide costs are estimated at 70 to 75 billion US dollars to maintain the renal replacement therapy of the roughly 1.1 million worldwide dialysis patients in 2001. In Ghana, renal replacement therapy (mainly haemodialysis and peritoneal dialysis) is available only in two of the teaching hospitals, and the estimated cost of dialysis is GHC 57,600per patient per annum. This amount is rather high for a country with a per capita income of 
$\$ 1500$ and a GDP of $6.3 \%$. The first renal transplant in this country was performed at the end of 2008 by a combined team of Ghanaian and British surgeons. Moreover, the number of patients requiring renal replacement therapy is increasing globally, by up to $7 \%$ annually according to some reports (Gansevoort et al., 2004; Jones et al., 2005; Muntner et al., 2005).

\section{CHRONIC KIDNEY DISEASE}

Chronic kidney disease (CKD) affects around 10$13 \%$ of the general population, with only a small proportion in end stage renal disease (ESRD), either on dialysis or awaiting renal transplantation. It is well documented that CKD patients have an extremely high risk of developing cardiovascular disease (CVD) compared with the general population, so much so that in the early stages of CKD patients are more likely to develop CVD than Chronic kidney disease (CKD) comprises either glomerular filtration rate (GFR) $<60 \mathrm{ml} / \mathrm{min} / 1.73 \mathrm{~m}^{2}$ for more than 3 months, or other pathological abnormalities or markers of kidney damage, including abnormalities in blood, urine tests or imaging studies. Detection of pre-renal disease and CKD stages 1 and 2 cannot therefore rely on measurement of GFR alone and instead require assessment of persistent proteinuria, microalbuminuria and haematuria, alongside eGFR levels derived from serum creatinine. Using these parameters, around $10 \%$ of the UK population have early CKD (pre-renal, stages 1, 2 and 3) and of these $40 \%$ are in CKD stage 3.In the , approximately $13 \%$ of the population have CKD stage 3 and 4, accounting for $4.5 \%$ of the total population, and with half a million in end stage renal disease (ESRD); the comparison between NHANES II and III revealing a rise in the incidence of CKD in the US. ${ }^{[1,3]}$ There is a major further projected rise in the incidence of ESRD, and thus dialysis-requiring kidney failure, from the major increases in prevalence of obesity, type 2 diabetes and increases in population longevity, which, unless addressed, will threaten to overwhelm healthcare systems in the future.

Patients with CKD have a markedly increased incidence of cardiovascular events and cardiovascular disease (CVD) mortality compared with age-matched counterparts in the general population examined a large Medicare database of 1,091,201 patients to investigate the incidence atherosclerotic vascular disease, congestive heart failure, RRT and death. Individuals with early CKD were much more likely to die than reach renal replacement therapy (RRT), with rates per 100 patient years of 17.7 and 1.6 respectively. This is compared with the rate of 35.7 with atherosclerotic vascular disease and 30.7 with congestive heart failure, illustrating the extreme susceptibility of these individuals to CVD and premature death. These findings were further supported in a study of 27,998 patients with early stages of CKD followed up over 5 years to investigate rate of progression to RRT and mortality. In those individuals with CKD stages 2 and 3,1.1\% and 1.3\% required dialysis, while the mortality rate was $19.5 \%$ and $24.3 \%$ respectively. There is therefore unequivocal evidence that subjects with CKD are among the highest-risk cohorts for cardiovascular events of any studied population. Current cardiovascular risk prediction charts do not adequately incorporate this profound additional risk in calculating 10-year event rates, leading to underestimations and suboptimal primary prevention measures in patients with CKD.

While recent data and consensus have challenged the position of diabetes mellitus as a true coronary heart disease risk equivalent, a growing body of evidence supports this degree of risk in CKD they are to progress to ESRD. Various pathophysiological pathways and explanations have been advanced and suggested to account for this, including endothelial dysfunction, dyslipidaemia, inflammation, left ventricular hypertrophy and cardiac autonomic dysfunction. In this review, we try to understand and further explore the link between CKD and CVD, as well as offering interventional advice where available, while exposing the current lack of RCT-based research and trial evidence in this area Epidemiological research in kidney diseases was long hampered by lack of a common definition of chronic kidney disease (CKD). In 2002 the Kidney Disease Outcome Quality Initiative (K/DOQI) proposed a classification of CKD for diagnosis and risk stratification.

In end stage kidney disease (ESKD) patients, mortality due to cardiovascular disease (CVD) is 10 to 30 times higher than in the general population. For example, a 30-year-old dialysis patient has a similar CVD mortality risk to that of an 80-year-old in the general population. This increased risk likely reflects the very high prevalence of CVD, increased prevalence and severity of diabetes, hypertension and left ventricular hypertrophy, and non-traditional risk factors such as chronic volume overload, hypophosphatemia, anaemia, oxidant stress, and other 
aspects of the uremic milieu. In this chapter, we focus on epidemiology and management of traditional and non-traditional CVD risk factors, and on ischemic heart disease, heart failure, pericardial effusion, valvular disease, and arrhythmia.

Definition: -Structural and functional abnormalities of the kidney for $>3$ month as manifested by either Kidney damage, with or without deceased GFR as defined by:-

$>$ Pathologic abnormalities

$>$ Marker of kidney damage, including abnormalities in composition of blood in urine or abnormalities in imaging test.

Table 1: Definition of chronic kidney disease (CKD) stages according to the K/DOQI guidelines

\section{GFRml/min $/ 1.73 \mathrm{~m} 2$} Stages Description

1. Kidney damage with normal or increased GFR $\geq 90$

2. Kidney damage with mild reduction of GFR 60-89

3. Moderate reduction of GFR $30-50$

4. Severe reduction of GFR $15-29$

5. Kidney failure $<15$ or dialysis

Based on this classification increasing knowledge of the epidemiology and significance of chronic kidney disease have evolved. More prevalent hypertension, diabetes mellitus and obesity partly explained the increase. In addition to increasing age, these are all important risk factors for development and progression of CKD. This raises concern about future increased incidence of end stage renal disease (ESRD) and other complications of CKD including increased cardiovascular disease.

Cardiac disease is the major cause of death, accounting for 41 percent of all-cause mortality in patients receiving hemodialysis (Lafrance et al., 2006). Cardiac diseases are associated independently with a decrease in kidney function and progression of existing kidney diseases (Elsayed et al., 2007), In both the acute setting and more long-term phase, even small decreases in GFR are associated with adverse outcome (Coca et al., 2007).

Persons with CKD are predisposed to three types of CVD, atherosclerosis, arteriosclerosis, and cardiomyopathy when compared with age and gender matched persons with normal kidney function (Wail et al., 2005). In the past cardiovascular death was mainly viewed as the result of accelerated coronary heart disease (CHD). Although CHD is undoubtedly more frequent than in the background population, the importance of the two other, largely unresolved cardiovascular problems, Sudden death and cardiomyopathy (Remppis and Ritz., 2008).

\section{Table (1): Risk Factors for Cardiovascular} Disease in Kidney Disease

\section{Traditional Risk Factors}

$>$ Age sex

$>$ Diabetes mellitus

$>$ Hypertension

$>$ Smoking

$>$ Dyslipidemia

$>$ Family history

$>$ Obesity

Nontraditional Risk Factors

Kidney function decline

$>$ Albuminuria

$>$ Anaemia

$>$ Inflammation and oxidative stress

$>$ Disorders of mineral metabolism

- Hyperphosphatemia

- Changes in vitamin

D metabolism

Secondary hyperparathyroidism

- Elevated FGF-23 levels (fibroblast growth factor

23)

Activation of the sympathetic nervous system

Cardio renal syndrome:-

Patients with ESRD are well known to suffer a high risk of cardiovascular morbidity and mortality, having more than 10-fold increased risk of cardiac death than age-/gender-matched controls in the general population. Cardiovascular risk is shown to increase even in early stages of CKD. Death due to cardiovascular disease is much more common in patients within all stages of CKD than reaching ESRD needing renal replacement therapy). Multiple explanations may exist for the high cardiovascular risk in CKD patients.

Traditional risk factors for cardiovascular disease, such as hypertension, diabetes mellitus, smoking and dyslipidaemia are risk factors for CKD as well, and highly prevalent in this population. In the course of declining renal function, kidney specific risk factors for cardiovascular disease like accumulation of uremic toxins, inflammation, anaemia, disturbances in calcium and phosphorous balance, lack of active vitamin $\mathrm{D}$ and sodium and water retention among others, will contribute to left ventricular hypertrophy 
$(\mathrm{LVH}), \quad$ chronic inflammation, accelerated atherosclerosis, extra vascular calcifications and endothelial dysfunction. CKD patients are also less likely to receive risk-modifying medication and interventions compared to non-CKD patients. Just like cardiovascular disease is prevalent in patients with renal disease, primary heart conditions are commonly associated with renal dysfunction. Reduced renal perfusion, often predisposed by micro vascular and macro vascular disease in the context of the same vascular risk factors associated with cardiovascular disease, may hemodynamically affect kidney function in HF patients. Furthermore, neurohormonal activation, renal venous congestion and adverse effects of pharmacotherapies used in the management of HF, have been suggested as causes for the high prevalence of renal dysfunction in $\mathrm{HF}$ patients. Regardless the cause, impaired and worsening, renal function in patients with acute and chronic HF is associated with adverse outcomes and prolonged hospitalization. Even slight decrease in GFR is found to significantly increase mortality risk in patients with chronic HF. Combined heart and kidney dysfunction is common. Primary disorders of either kidney or heart often result in secondary dysfunction or injury to the other organ.

The cardiorenal syndrome (CRS) was earlier used to describe a relatively normal kidney that is dysfunctional because of a diseased heart, with the assumption that in the presence of a healthy heart, the same kidney would perform normally. The more recent recognition of the numerous negative effects of heart disease on kidney function and kidney disease on heart function led to a new definition of the CRS, dividing into 5 different subtypes ofcardiorenal syndrome .The present study included patients with chronic HF and thereby investigates topics related to cardiorenal syndrome type 2. Impaired kidney function in patients with chronic $\mathrm{HF}$ is associated with adverse prognosis and even slight decreases in estimated GFR (eGFR) significantly increase mortality risk.

Much of our knowledge on the relationship between $\mathrm{HF}$ and renal function originate from selected patient populations in clinical trials or hospitalized patients. There is limited understanding of the pathophysiology of renal dysfunction even in advanced heart failure. Hemodynamic issues as venous congestion and reduced renal perfusion due to powerful vasoconstriction are considered important. However, neurohormonal activation including the RAAS and SNS, oxidative stress, comorbidities and pharmacological heart failure treatment have a role as cardiorenal connectors affecting renal function. The explanation for the impaired prognosis observed in patients with chronic HF and CKD is also incompletely understood. A higher burden of comorbidities, more severe vascular disease and worsened cardiac function may explain part of the alteration.

Furthermore, large randomized trials that have shaped the treatment of chronic HF in the two last decades, have consistently excluded patients with significant renal disease. Lack of evidence based clinical treatment may cause that patients with chronic HF and renal dysfunction are less likely to receive potential life-saving treatment, thereby altering prognosis. 


\section{Definition of cardiorenal syndrome (CRS):-}

\begin{tabular}{|l|l|}
\hline $\begin{array}{l}\text { Cardiorenal syndrome } \\
\text { (CRS) general definition }\end{array}$ & $\begin{array}{l}\text { general definition } \\
\text { A complex pathophysiological disorder of the heart and the kidneys whereby } \\
\text { acute or chronic dysfunction in one organ may induce acute or chronic } \\
\text { dysfunction in the other organ. }\end{array}$ \\
\hline $\begin{array}{l}\text { CRS type 1 (acute } \\
\text { cardiorenal syndrome) }\end{array}$ & $\begin{array}{l}\text { Abrupt worsening of cardiac function (e.g. acute cardiogenic shock or acute } \\
\text { decompensation of chronic heart failure) leading to kidney injury. }\end{array}$ \\
\hline $\begin{array}{l}\text { CRS type 2 (chronic } \\
\text { cardiorenal syndrome) }\end{array}$ & $\begin{array}{l}\text { (chronic cardiorenal syndrome) } \\
\text { Chronic abnormalities in cardiac function (e.g. chronic heart failure) causing } \\
\text { progressive chronic kidney disease. }\end{array}$ \\
\hline $\begin{array}{l}\text { CRS type 3 (acute } \\
\text { renocardiac syndrome) }\end{array}$ & $\begin{array}{l}\text { Abrupt worsening of renal function (e.g. acute kidney failure or } \\
\text { glomerulonephritis) causing acute cardiac disorder (e.g. heart failure, } \\
\text { arrhythmia, pulmonary edema). }\end{array}$ \\
\hline $\begin{array}{l}\text { CRS type 4 (chronic } \\
\text { renocardiac syndrome) }\end{array}$ & $\begin{array}{l}\text { (chronic renocardiac syndrome) } \\
\text { Chronic kidney disease (e.g. chronic glomerular disease) contributing to } \\
\text { decreased cardiac function, cardiac hypertrophy and/or increased risk of } \\
\text { adverse cardiovascular events. }\end{array}$ \\
\hline $\begin{array}{l}\text { CRS type 5 (secondary } \\
\text { cardiorenal syndrome) }\end{array}$ & $\begin{array}{l}\text { Systemic condition (e.g. diabetes mellitus, sepsis) causing both cardiac and } \\
\text { renal dysfunction. }\end{array}$ \\
\hline
\end{tabular}

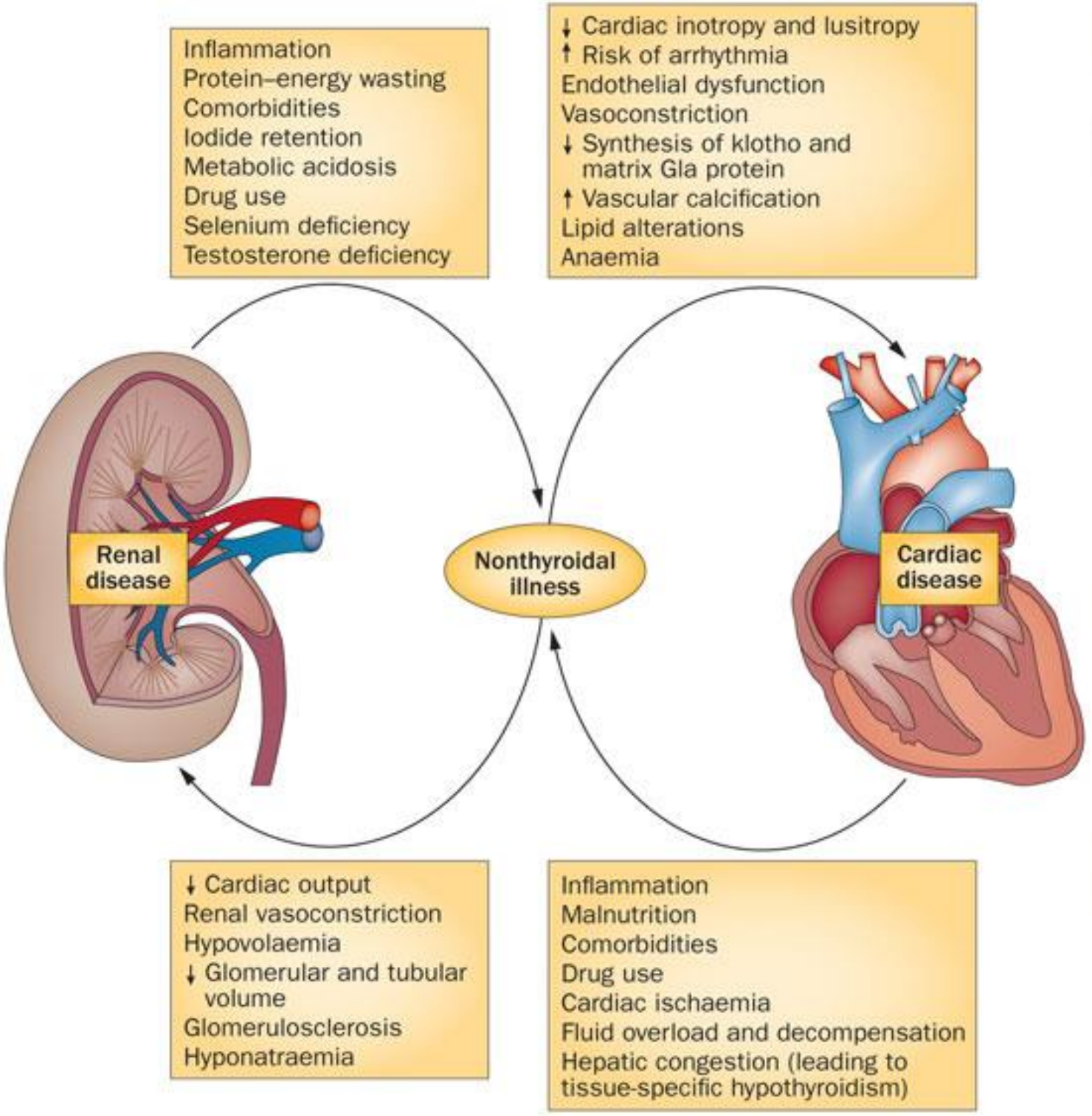

Figure showing:-Cardio renal syndrome 


\section{Uremic cardiomyopathy:-}

In chronic uraemia, cardiomyopathy may manifest as concentric LVH, or left ventricular dilatation, and may result in diastolic or systolic dysfunction. These disorders are associated with the subsequent development of cardiac failure and with death (Parfrey and Foley, 2005).

\section{Concentric LVH:-}

Left ventricular hypertrophy (LVH) is a common finding in mild renal disease and end-stage renal disease, with some claiming an incidence of nearly 75 to 80 percent in dialysis patients (Stewart et al., 2005).

Left ventricular enlargement is very common at the starting of dialysis therapy, and highly predictive of future cardiac morbidity. It is not known whether cardiac size increases further while on dialysis therapy and whether potentially reversible risk factors for later progression can be identified (Aoki et al., 2005).

\section{Myocardial fibrosis:-}

Myocardial fibrosis is not an obligatory consequence of hemodynamic stress and is more marked in pressure overload than in volume overload. The causes of myocardial fibrosis are multifactorial and include ischemia, and effects of hormones such as catecholamine's, angiotensin $\Pi$ and aldosterone. Other studies have demonstrated that parathyroid hormone is a permissive factor in the genesis of cardiac interstitial fibrosis (London, 2002).

\section{Systolic dysfunction:-}

Decreased systolic function is observed frequently in patients with cardiac diseases pre-existing before ESRD therapy or in patients with sustained and marked hemodynamic overload. Diminished myocardial contractility may be a result of overload cardiomyopathy (Parfrey and Foley, 2005).

\section{Diastolic dysfunction:-}

Diastolic filling is frequently altered in dialysis patients. The abnormal ventricular filling in ESRD results from increased LV stiffness caused by intramyocardial fibrosis and delayed relaxation. It is highly likely that patients with concentric LVH or LV dilatation have diastolic dysfunction, which predisposes to the development of heart failure (Parfrey and Foley, 2005).

\section{Rhythm disturbance:-}

Hemodialysis patients have a rate of arrhythmias that is 40 times greater than the general population, but the causes and types of fatal arrhythmias are still unclear, Dialysis increases the arrhythmogenic activity with respect to the inter-dialysis period to a great extent (Al- Khatib et al., 2007).

Abnormalities on resting electrocardiography are common in dialysis patients. Among patients who were enrolled in the $4 \mathrm{D}$ study $(\mathrm{n}=50), 11 \%$ had a rhythm other than sinus; three of four patients with an alternative rhythm had atrial fibrillation (Krane et al., 2009).

Most clinicians have been reluctant to administer prophylactic anticoagulation therapy for chronic AF in dialysis patients. Furthermore, the benefits versus risks from anticoagulation have not been accurately determined in these patients (Genovesi et al., 2009).

Dispersion of the QT interval has emerged as an important predictor of ventricular arrhythmia. The QT dispersion is simply the difference between the shortest and longest QT interval on a standard surface. This is a non-invasive measurement of myocardial repolarization in homogeneity and hence predisposition to re-entry arrhythmias. A QT dispersion above $80 \mathrm{Ms}$ reflects a loss of synchronization in the repolarization process. The QT interval is partially influenced by the concentration of the dialyzable cations calcium, magnesium and potassium and may also be influenced by cardiac filling pressures (Fukuta et al.)

To prevent haemodialysis-related myocardial arrhythmias, prolonged dialysis sessions with low ultrafiltration rates, Careful titration of target weight and administration of oxygen during dialysis are recommended. Additionally, beta blockers seem to be an important therapeutic option in high risk patients (Fukuta $\mathrm{H}$ et al., 2003).

Hemodialysis patients administered on digitalis therapy have increased risk of arrhythmia. Also duration of hemodialysis, and acetate containing dialysate have close relation with occurrence of arrhythmias among haemodialysis patients. A high calcium phosphate product predialysis may be correlated with increased incidence of ventricular arrhythmias (Antonio et al., 2008). 
Atherosclerotic disease:-

Because of the high prevalence of hypertension, LVH, diabetes, and lipid abnormalities in dialysis patients, it has been suggested that these patients have an accelerated rate of coronary atherogenesis. atherosclerotic disease in CKD and in dialysis patients is somewhat different from that in the general population with atherosclerosis, as this atherosclerotic burden is further complicated by an increased frequency of calcific lesions, an increase in medial thickness and calcification involving medium to large sized vessels (Wali et al., 2005).

Multiple factors contribute to the vascular pathology of chronic uraemia, including injury to the vessel wall, prothrombotic factors, lipoprotein interactions, proliferation of smooth muscle, hyperhomocysteinemia, increased oxidant stress, and diminished antioxidant levels (Wali et al., 2005).

\section{Congestive heart failure:-}

Not surprisingly, the presence of heart failure independently predicts early mortality in end-stage renal disease as it does in nonuremic patients (Postorino et al., 2007).Congestive heart failure (CHF) may result from systolic failure, usually caused by dilated cardiomyopathy, or from diastolic dysfunction, usually caused by LVH. In fact diastolic dysfunction is almost as frequent cause of recurrent or persistent $\mathrm{CHF}$ in dialysis patients as is dilated cardiomyopathy (Aoki et al., 2005).

Among patients with diastolic dysfunction, $\mathrm{CHF}$ results from impaired ventricular relaxation, which leads to an exaggerated increase in LV end-diastolic pressure for a given increase in $\mathrm{LV}$ end diastolic volume. As a result, a relatively small excess of salt and water intake leads rapidly to a large increase LV end diastolic pressure, culminating in the pulmonary edema (Parfrey and Foley, 2005).

\section{Ischemic heart disease :-}

Evaluation for coronary artery disease should be performed in dialysis patients with symptoms and/or signs of coronary artery disease, a change in symptoms and signs, including recurrent hypotension, heart failure that is unresponsive to changes in dry weight, and intradialytic hypotension that prevents attaining dry weight (Gill JS et al., 2005).
The K/DOQI guidelines recommend that, at initiation of dialysis, all patients should undergo baseline echocardiography and electrocardiography (K/DOQI, 2005).

\section{Anaemia in heart failure:-}

Chronic heart failure is a syndrome with various pathophysiological consequences. Neuroendocrine, metabolic and immunological changes are identified as HF develops. Anaemia has been recognized by clinicians in HF patients for decades, but has only recent years received attention with systematic research. $\mathrm{CKD}$ is a common comorbidity in $\mathrm{HF}$ patients with anaemia. The kidneys are essential in the endogenous production of erythropoietin, and renal dysfunction may induce anemia in HF patients creating a vicious circle, termed the "cardio-renalanaemia syndrome". In addition to renal dysfunction, anaemia of chronic disease, iron-deficiency and hem dilution appears to be important aetiologies of anaemia in HF patients). Treatment with ACE and ARB may also contribute to anaemia as these agents may inhibit erythropoiesis. Depending on the definition used and population studied, the prevalence of anaemia in HF patients varies from 9 to $69 \%$ ).

Anaemia is identified as an independent prognostic factor of mortality in $\mathrm{HF}$ patients and haemoglobin levels are related to symptoms and quality of life. Increased myocardial workload due to hemodynamic and neurohormonal changes is a consequence of anaemia, thereby inducing cardiac remodelling and left ventricular hypertrophy $(\mathrm{LVH})$. This may partly explain the impaired prognosis of anaemia in $\mathrm{HF}$ patients. Clinical trials investigating specific anaemia treatment with erythropoiesis stimulating agents (ESA) and intravenous iron in HF patients with anaemia have been promising in regard to symptoms and quality of life. Convincing data on mortality are missing and we do not yet know when to start anaemia treatment, treatment goals and who will benefit from treatment. Actually, we don't know if anaemia is a mediator or just a marker of poor prognosis in patients with chronic heart failure. 


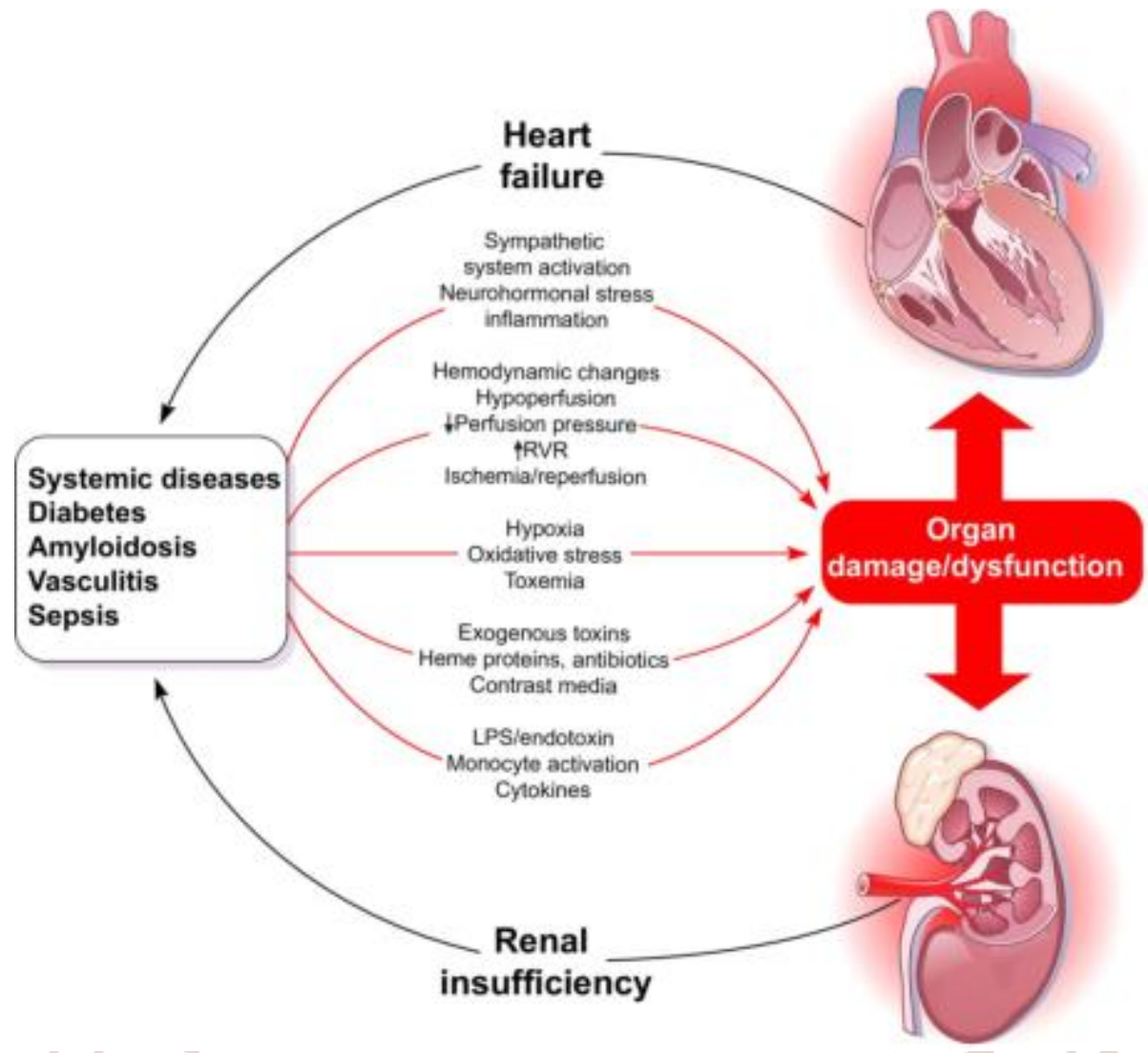

Figure showing :- anaemia in heart failure

\section{AIMS OF THE STUDY}

The overall aim of the present study is to investigate the association between various risk factors of CVD and different stages of CKD in patients with kidney replacement therapy

\subsection{The specific objective are:-}

To classify the CKD patients into 5 stages according to GFR and albuminuria.

$>$ To correlate laboratory findings with CKD classes.

$>$ To determine the main cardiovascular risk factors in the patients.

$>$ To study the relationship between CKD progression and development of CVD.

\subsection{Significance:-}

$>$ This study is carried out for the CKD in haemodialysis patients .

$>$ Identification of CVD risk factors among patients with $\mathrm{CKD}$ is potentially useful for raising awareness of the relationship between CKD and CVD.

$>$ Encouragement of clinicians to evaluate their $\mathrm{CKD}$ patients for $\mathrm{CV}$ risk factors, such risk factors may be used in monitoring the progression of the disease and predicting the future events.
Initiation of treatment for $\mathrm{CV}$ risk factors at earlier stages of CKD, which may be effective in reducing CVD events both before and after the onset of kidney failure.

\section{MATERIALS AND METHODS}

\subsection{Study design:-}

The present study is a cross sectional study, that was conducted from the beginning of March 2016 to the end of august, 2016.

\subsection{Setting of the study:-}

The study was carried out at the Nephrology Department of Dr B. R. A.M. HOSPITAL RAIPUR.

\subsection{Population of the study:-}

Patients from both genders who had CKD on Hemodialysis patient and registered in the Nephrology Department of Dr. B. R. A. M. HOSPITAL RAIPUR. The total number of cases in the department was 50 patients at the end of the study.

\subsection{Study sample:-}

Study sample comprised 50 subjects $\mathrm{CKD}$ on $\mathrm{HD}$ patients. 
International Journal of Trend in Scientific Research and Development (IJTSRD) ISSN: 2456-6470

\subsection{Selection criteria:-}

\subsubsection{The inclusion criteria}

$>$ Patients with age $>17$ years old.

$>$ Diagnosed as CKD patients on HD

\subsubsection{The exclusion criteria}

$>$ Patients with kidney replacement therapy haemodialysis,

$>$ Clinically overt inflammatory disease at the time of investigation.

$>$ Clinically significant over hydration or dehydration.

$>$ Patients on anti-lipid drugs.

\subsection{Ethical considerations}

1. Coordination with the Ministry of Health was fulfilled

2. Patient of the participants were given a full explanation about the purpose of the study, assurance about the confidentiality of the information obtained through the questionnaire and blood analysis, and that they have the right to refuse to participate or to drop out in any phase of the study.

\subsection{Data Collection}

Data were collected by both direct and indirect methods.

\subsubsection{The direct method}

Laboratory investigations including blood sampling after 12-14 hour overnight fasting and the following blood tests were done for patients and controls: Creatinine, urea, , cholesterol, triglycerides, HDL, LDL, , haemoglobin, . Additionally, spot urine samples were tested for ACR. Glomerular filtration rate was estimated in all participating subjects according to the Schwartz formula. , weight, blood pressure were measured for each participant. Body mass index was also calculated for all subjects.

\subsubsection{The indirect method}

Face to face interview questionnaire was administrated for both patients and controls (Appendix 1), the questionnaire main content included:

1. Personal data.

2. History of the disease (for patients)

3. Physical activity

4. History of CVD in the patients, family

\subsection{Statistical analyses}

Data entry and statistical analyses were performed using SPSS (Statistical Package for Social Sciences) Results were expressed as frequency or Mean \pm SD.

\subsection{Materials}

Parameters of clinical study:-

50patients (34 males and 16 females with mean age 48.4249years) with CKD and dialyzed 50 long-term HD patients, were included in this one centre study. 50 patients of CKD patients yetdialyzed, the same group of HD patients as in the first study was used and no any healthy subjects were studied. Patients at different stages of CKD were included in a crosssectional study. Patients were separated into five CKD groups according to their estimated glomerular filtration rate [eGFR; Modification of Diet in Renal Disease (MDRD)], based on Kidney Dialysis Outcomes Quality Initiative (K/DOQI), as follows:

stage $5(\mathrm{n}=49 ;$ eGFR $<15 \mathrm{ml} / \mathrm{min})$,

stage $4(\mathrm{n}=1$; eGFR $15-30 \mathrm{~mL} / \mathrm{min})$,

stage $3(\mathrm{n}=0 ; 30-60 \mathrm{~mL} / \mathrm{min})$.

stage 1 and 2 were analysed together $(n=0 ; G F R>60$ $\mathrm{mL} / \mathrm{min}$ ).

Blood tests and physiological parameters were obtained for each patient at the time of enrolment but before initiation of RRT. Theaetiologies of AKI were ischemia (39.8\%), nephrotoxicity (22\%), and multifaceted factors $(38.2 \%)$. 42 patients with CKD stage 5 with glomerular filtration rate (eGFR $<15$ $\mathrm{ml} / \mathrm{min} / 1.73 \mathrm{~m} 2$ ) at the onset of RRT were included. The aetiology of CKD was vasculits $(11 \%)$, chronic glomerulonephritis (23\%) hypertension $(19 \%)$ and diabetes $(12 \%)$. The same group of HD patients as in the first and second study was used for comparison. Written informed consent and laboratory samples were obtained from all subjects according to ethical guidelines. The study was performed in adherence to the principles of the Declaration of DR. B. R. A. M. HOSPITAL RAIPUR and approved by the Institutional Ethical Committee. 


\subsubsection{Chemicals and reagents}

Chemicals and reagents used in this study are shown in Table 3.9.1.

\section{Reagent}

Urea
Creatinine
CBC
TLC
DLC
Microalbumin (urine)
Cholesterol
Triglycerides
HDL-

\subsubsection{Equipment}

The main equipment that were used are listed in Table 3.2 .

\begin{tabular}{|l|}
\hline \multicolumn{2}{|c|}{ Instrument } \\
\hline Spectrophotometer \\
Echo machine \\
ECG Machine \\
Electrolyte analyser \\
Blood Counter \\
Centrifuge \\
Refrigerator with Freezer -20C \\
Water Bath \\
Vortex Mixer \\
Micropipettes
\end{tabular}

\subsection{Methods}

\subsubsection{Sampling and processing:-}

Blood samples were collected from patients and controls who agreed to participate in the study after overnight 12- $14 \mathrm{hr}$. Five $\mathrm{ml}$ blood were obtained from each subject and divided into EDTA tube (1.0 $\mathrm{ml})$ and vacationer plain tube $(4.0 \mathrm{ml})$. Vacationer plain tubes were left for short time to allow blood to clot, then clear serum samples were obtained by centrifugation at $4000 \mathrm{rpm}$ for 10 minutes. A spot sample of morning urine in the fasting state was collected from each subject. Urine albumin, urine creatinine and $\mathrm{CBC}$ were done in the same day of collection. Serum samples were stored at $-20{ }^{\circ} \mathrm{C}$ until the time of performing the analysis. All biochemical analyses were done in the laboratory of the Dr. B.R.A.M.HOSPITAL RAIPUR.

\subsubsection{Blood Pressure: -}

Blood pressure was measured by trained personnel using a mercury sphygmomanometer and a stethoscope. Measurements were taken from the left upper arm after subjects had been sitting for $>5 \mathrm{~min}$ in accordance with the recommendations of the American Heart Association (Kirkendall et al., 1967). Duplicate measurements were taken with a 5 minute rest interval between measurements and the mean value was recorded to the nearest $2.0 \mathrm{mmHg}$

\subsubsection{URINALYSIS:-}

Early morning urine was collected in plastic containers from the respondents and urine protein was determined using the dip-stick qualitative method (CYBOW'M DFI Co Ltd, Gimhae-City, Republic of Korea).

\section{Principle}

The test is based on the protein error of indicators principle. When $\mathrm{pH}$ is held constant by a buffer indicator, dyes release $\mathrm{H}+$ ions because of the protein present and change colour from yellow to blue-green.

\subsubsection{SAMPLE COLLECTION PREPARATION :-}

AND

Venous blood samples were collected after an overnight fast (12-16 hours). About $7 \mathrm{mls}$ of venous blood were collected and, $5 \mathrm{ml}$ dispensed into vacutainer ${ }^{\circledR}$ plain tubes. After clotting, it was then centrifuged at $500 \mathrm{~g}$ for $15 \mathrm{~min}$. The serum was stored at $-80^{\circ} \mathrm{C}$ until assayed. The remaining $2 \mathrm{ml}$ were dispensed into tubes containing $2.5 \mu \mathrm{g}$ of potassium ethylenediaminetetraacetic acid (K2 EDTA) as an anticoagulant.

\section{Biochemical assays}

Serum biochemistry was performed on theDR B.R.A. Hospital Raipur. renal function tests - serum sodium $(\mathrm{Na}+)$, serum potassium $(\mathrm{K}+)$, blood urea nitrogen (BUN), serum creatinine (CRT), serum uric acid; electrolytes - serum calcium (Ca2+), serum magnesium ( $\mathrm{Mg} 2+)$ and serum phosphate (PO43-). Also lipid profile which include total cholesterol (TC), triglycerides (TG), high density lipoprotein cholesterol (HDL-C), very low density lipoprotein cholesterol (VLDL-C), low density lipoprotein cholesterol (LDL-C) and coronary risk were determined.

\section{Cholesterol}

\section{$>$ Principle}

The present method utilizes a phenol substitute (4aminoantipyrine (4-AAP) that performs like phenol but without being corrosive. The intensity of the red colour produced is directly proportional to the total cholesterol in the sample when read at $500 \mathrm{~nm}$. 


\section{Triglycerides}

\section{$>$ Principle}

The present method uses a modified Trinder (Trinder, 1969; Barham and Trinder, 1972) colour reaction to produce a fast, linear, endpoint reaction. Triglycerides in the sample are hydrolysed by lipase to glycerol and fatty acids. The glycerol is then phosphorylated by ATP to glycerol-3-phosphate (G3P) and ADP in a reaction catalysed by glycerol kinas. G3P is then converted to dihydroxyacetone phosphate (DAP) and hydrogen peroxide by glycerophosphate oxidise (GPO). The hydrogen peroxide then reacts with 4-aminoantipyrine (4-AAP) and 3, 5-dichloro-2-hydroxybenzen (3,5-DHBS) in a reaction catalyzed by peroxidise to yield a red coloured quinoneimine dye. The intensity of the colour produced is directly proportional to the concentration of triglycerides in the sample.

\section{HDL-Cholesterol}

\section{$>$ Principle}

The method employed herein is in a two reagent format. The first reagent contains anti human $\beta$ lipoprotein antibody which bind to lipoproteins (LDL, VLDL and chylomicrones) other than HDL. The second reagent contains enzymes which then selectively react with the cholesterol present in the HDL particles. Consequently only HDL cholesterol is subject to cholesterol measurement. The primary reading is done at $600 \mathrm{~nm}$ and the secondary at 700 $\mathrm{nm}$.

\section{Urea Nitrogen (BUN)}

Determination of urea nitrogen in serum is widely used as a screening test for renal function. When used in conjunction with the determination of creatinine in serum, it is helpful in the differential diagnosis of the three types of azotemia; pre-renal, renal and postrenal.

\section{$>$ Principle}

The present procedure is based on a modification of the method of Talked and Schubert (1965). Urea is hydrolyzed in the presence of water and urease to produce ammonia and carbon dioxide. The liberated ammonia reacts with $\alpha$-ketoglutarate in the presence of NADH to yield glutamate. An equimolar quantity of NADH undergoes oxidation during the reaction catalyzed by Glutamate dehydrogenase (GLDH) resulting in a decrease in absorbance $(340 \mathrm{~nm})$ that is directly proportional to the urea nitrogen concentration in the sample.

\section{Creatinine}

Creatinine measurements are used in the assessment of renal dysfunction. Elevated creatinine levels are found in renal diseases and insufficiency with decreased glomerular filtration (uraemia or azotemia if severe); urinary tract obstruction; reduced renal blood flow including congestive heart failure, shock and dehydration.

\section{$>$ Principle}

This method is based on a modification of the kinetic procedure which is fast, simple and avoids interferences incorporating a surfactant and other ingredients to minimize protein and carbohydrate interferences. Creatinine reacts with picric acid in alkaline conditions to form a colour complex (yelloworange) which absorbs at $510 \mathrm{~nm}$. The rate of formation of colour is proportional to the creatinine in the sample.(Fabiny and Ertingshausen, 1971)

\section{Biological Activities}

Human parathyroid hormone (hPTH) is a major physiological regulator of phosphocalcic metabolism. hPTH increases serum calcium concentration by its actions Un akidney (enhancing tubular $\mathrm{Ca} 2+$ reabsorption and phosphate excretion) and bone (Stimulating osteoclastic activity and bone (stimulating osteoclastic activity and bone resorption). It indirectly affects intestinal absorption of $\mathrm{Ca} 2+$ by stimulating renal $1 \alpha$-hydroxylation of 25 hydroxyvitamin D. The release of PTH is controlled in a negative feedback loop by the serum concentration of $\mathrm{Ca} 2+$. PTH is synthesized in the chief cells of the parathyroid glands and secreted as an 84 amino acid moleule called " intact PTH " which is the main bioactive product. This molecule is degraded by proteolytic cleavage between amino acids 33-37 at peripheral site to form biologically active amino terminal fragments which are cleared only by glomerular filtration, while the bioactive intact PTH and amino- terminal fragments are also metabolically degraded in the liver and other tissues. Thus the measurement of intact PTH correlates best with the hormone production and biological activity.

\section{Principles}

The GenWay hPTH-EASIA is a solid phase Enzyme Amplified Sensitivity Immunoassay performed on microtitre plates. Calibrator and samples react with the captured polyclonal antibodies (PAb, goat anti 134 PTH fragment) coated on microtitre well. After incubation, the excess of antigen is removed by washing. The monoclonal antibodies labeled with 
horse radish peroxidase (HRP) are added. After an incubation allowing for the information of a sandwich: coated PAbs-human PTH-Mab - HRP the microtitre plate is washed to remove unbound enzyme labeled antibody. Bound enzyme- labeled anti body is measured through a chromogenic reaction. The chromogenic solution tetramethyl benzydine (TMB) is added and incubated. The reaction is stopped with the addition of stop solution and the microtitre plate is then read at the appropriate wavelength.

\subsubsection{Calculated measurements :-}

Glomerular filtration rate was calculated by MDRD fomula: GFR ( $\mathrm{ml} / \mathrm{min} / 1.73 \mathrm{~m} 2)$

$>$ Albumin/creatinine ratio $\mathrm{ACR}(\mathrm{mg} / \mathrm{g})=$ Microalbumin in urine $(\mathrm{mg} / \mathrm{L}) \times 1000$ /creatinine in urine $(\mathrm{mg} / \mathrm{dl}) \times 10$ the urine creatinine value is multiply by 10 to convert $\mathrm{mg} / \mathrm{dL}$ to $\mathrm{mg} / \mathrm{L}$, then divide the urine albumin value by the urine creatinine value to arrive at the ratio, then multiply by 1000 to express the value as (mg albumin/g creatinine).

$>$ Low density lipoprotein was calculated by Friedewald equation: $\mathrm{LDL}(\mathrm{mg} / \mathrm{dl})=$ cholesterol (HDL + triglycerides)

\subsubsection{Biochemical analysis}

Serum urea, creatinine, albumin, cholesterol, triglycerides, HDL, urine albumin, and urine creatinine were analyzed manually using Stat Fax1904 plus spectrophotometer. Two levels of lyophilized multi-control sera ; normal and abnormal levels were analyzed with each run. The concentration of LDL was calculated from the results of a profile including total cholesterol, HDL and triglycerides using the Friedewald equation (LDL = cholesterol $(\mathrm{HDL}+$ triglycerides/5)) (Kaplan and Szabo, 1983). Biomedical,. Complete blood count was measured using Cell-DYN 1800 cell counter,

\section{REVIEW OF LITRETURE}

\subsection{The Kidneys}

\subsubsection{Location and structure}

The kidneys lie against the dorsal body wall beneath the parietal peritoneum in superior lumbar region where they receive some protection from the lower part of the rib cage. The right kidney is positioned slightly lower than the left kidney. Each kidney (about $12.5 \mathrm{~cm}$ long, $7.5 \mathrm{~cm}$ wide and $2.5 \mathrm{~cm}$ thick) has a medial indentation (the hilus) in which there is two renal arteries, renal vein, and ureter. A fibrous renal capsule encloses each kidney.

The kidney has three regions, outer granulated layer called renal cortex, renal medulla that consists of cone shaped tissue masses called medullary pyramids, and renal pelvis which is a central space or cavity that is continuous with the ureter (Marieb, 2003).

LOCATION- the kidney occupy the epigastria, hypochondric, lumber, umbilical region. Vertically they extend from the upper border of $12^{\text {th }}$ thorasicvertibra to the centre of body of $3^{\text {rd }}$ lumber vertebra. The right kidney is slightly lower than the left and the left kidney is a little nearer to the median plane than right.

The trans pyloric plane passes through the upper part of the hilus of the right kidney, and through the lower part of the hilus of the left kidney.

SHAPE SIZE WEIGHT - each kidney is about 11 $\mathrm{cm}$ long , $6 \mathrm{~cm}$ broad and $3 \mathrm{~cm}$ thick. The left kidney is a littlie longer and narrower than the right kidney .on an average the kidney weight $150 \mathrm{gm}$ in male and $135 \mathrm{gm}$ in female the kidney is raddish brown in colour. 

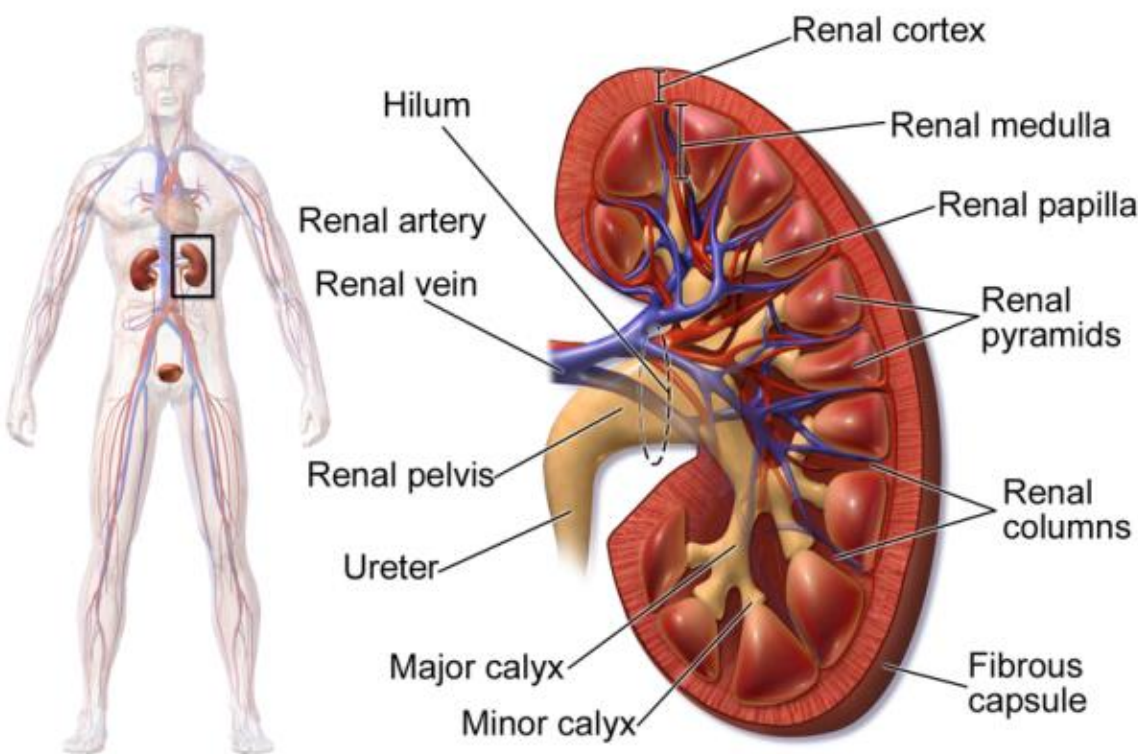

\section{Kidney Anatomy}

\section{Figure3.1. Location and structure of the kidney}

Each kidney contains approximately one million tiny structures called enthrones. Nephrons are responsible for the processes of filtration, reabsorption, and secretion that go on in the kidney to form the urine product. The nephron consists of two main structures, a glomerulus, which is a knot of capillaries, and a renal tubule. The closed end of the renal tubule is enlarged and cup-shaped and completely surrounds the glomerulus.
This portion of the renal tubule is called Bowman's capsule. In order from Bowman's capsule they are the proximal convoluted tubule, loop of Henle, and the distal convoluted tubule. Most of the nephron is located in the cortex, only portion of the loops of Henle dip into the medulla. Urine from many nephrons is collected in the collecting ducts, which deliver the final urine product into the calyces and pelvis of the kidney (Thibodeau and Patton, 1999)

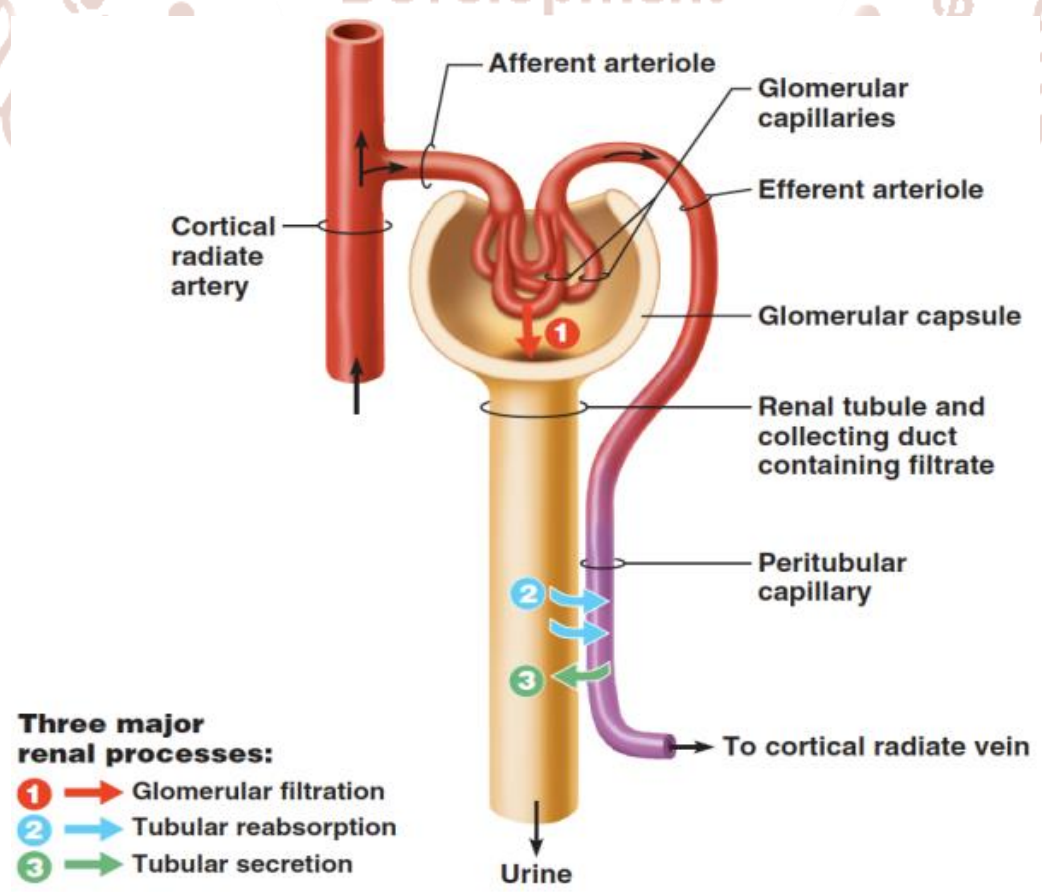

Figure3.2. Structure of the nephron 
Every nephon is associated with two capillary beds: The glomerulus and the peritubular capillary bed. The glomerulus is both fed and drained by arterioles. The afferent arteriole is the feeder vessel, and the efferent arteriole receives blood that has passed through the glomerulus. The efferent arterioles then breaks up to form the peritubular capillary bed, which closely clings to the whole length of the tubule. The peritubular capillaries then drain into an interlobular vein that leaves the cortex (Marieb, 2003).

\section{FUNCTIONS OF THE KIDNEYS:-}

The kidneys play an important role in the maintenance of normal body function. The basic function of the kidneys has to do with the formation of urine through complex filtration, reabsorption and secretion mechanisms. In addition, the kidneys also excrete urea and uric acid which are the end products of protein and nucleic acid metabolism.

The kidneys regulate fluid, electrolyte and acid base balance of the body and create a steady environment for the metabolic processes of tissues and cells. This function is essential for life and it is realized by balancing solute and water transport, excreting metabolic waste products, conserving nutrients, and regulating acid-base balance in the body.

Additionally, the kidney also produces three important hormones; erythropoietin which stimulates the production of red blood cells, renin which regulates blood pressure and calcitriol (the active form of vitamin D) which helps in regulation of plasma calcium levels.

\section{Principles of renal pathophysiology:-}

Renal injury can be characterized as either acute or chronic. Each has a distinctive clinical expression.

\subsection{Acute kidney injury}

Acute renal failure (ARF) is defined as "a rapid decline in renal filtration function". This condition is usually marked by a rise in serum creatinine concentration or azotemia (a rise in blood urea nitrogen concentration) immediately after a kidney injury.

Emergency dialysis may be needed until the situation resolves and the kidneys begin functioning again (Agraharkar, 2007). Acute renal insufficiency typically presents with the symptoms of volume overload secondary to impaired urine formation or excretion (Glassock, 1987).
The consequent retention of sodium and therefore of water can cause an expansion of the intravascular spaces and extravasation of fluid into the interstitial space throughout the body. The resulting volume expansion can therefore present as peripheral edema, pulmonary edema, or congestive heart failure. In acute renal failure both academia (resulting from failure to excrete or buffer the endogenous metabolic production of acids) and hyperkalemia (resulting from the lack of excretion of dietary potassium) can result in cardiac arrhythmias and sudden death. Acute uraemia has a particularly inhibitory effect on platelet function resulting in increase in the bleeding tendency (Kissane and Schreiner, 1990).

\subsection{Chronic kidney disease}

In chronic renal failure the metabolic consequences of uraemia are slowly progressive in nature. Chronic acidosis can affect myocardial contractility; contribute to central nervous system toxicity. Water and salt intake persistently exceeds excretory capacity, edema formation occurs. Chronic sodium retention can manifest as persistent arterial hypertension. Kidney also fails to convert 25 -hydroxyvitamin $\mathrm{D}$ to the metabolically active 1, 25- dihydroxyvitamin D, resulting in the defective absorption of calcium from the intestinal tract; secondary hypocalcemia induces secondary hyperparathyroidism with concomitant demineralization and resorption of bone. Depression of red cell production is the consequence of decreased renal production of the hormone erythropoietin. Increased red blood cell destruction resulting from uremic toxins as well as the mechanical damage to red cell observed in a variety of glomerular disease. Finally, patients with chronic kidney failure had depressed cellular immunity and humoral immunity (Kissane and Schreiner, 1990).

\section{CHRONIC KIDNEY DISEASE Definition of CKD:-}

The National Kidney Foundation Kidney Disease Outcomes Quality Initiative (NKF-KDOQI) in the USA defines CKD as "kidney damage for $\geq 3$ months, as confirmed by kidney biopsy or markers of kidney damage, with or without a decrease in GFR or GFR $<60 \mathrm{~mL} / \mathrm{min} / 1.73 \mathrm{~m} 2$ for $\geq 3$ months, with or without kidney damage".

Kidney damage is ascertained by either kidney biopsy or markers of kidney damage, such as urine abnormalities (proteinuria), blood abnormalities or abnormalities on imaging studies (NKF, 2002). 


\section{Glomerular filtration rate:-}

provides an excellent measure of the filtering capacity of the kidneys. A low or decreasing GFR is a good index of CKD. Since the total kidney GFR is equal to the sum of the filtration rates in each of the functioning nephrons, the total GFR can be used as an index of functioning renal mass (Rose, 1984)

\section{Table 4 .2 Prediction of glomerular filtration rate based on serum creatinine}

\begin{tabular}{|l|l|}
\hline \multicolumn{1}{|c|}{$\begin{array}{c}\text { Equation } \\
\text { Author }\end{array}$} & \multicolumn{1}{|c|}{ Equation } \\
\hline Schwartz & $\begin{array}{l}\text { GFR }\left(\mathrm{ml} / \mathrm{mim} / 1.73 \mathrm{~m}^{2}\right) \\
0.55 \times \text { length } / \mathrm{Scr}\end{array}$ \\
\hline $\begin{array}{l}\text { Counahan- } \\
\text { Barrat }\end{array}$ & $\begin{array}{l}\text { GFR }\left(\mathrm{ml} / \mathrm{mim} / 1.73 \mathrm{~m}^{2}\right)=0.43 \times \\
\text { length } / \mathrm{Scr}\end{array}$ \\
\hline $\begin{array}{l}\text { Abbreviated } \\
\text { MDRD Study }\end{array}$ & $\begin{array}{l}\text { GFR }\left(\mathrm{ml} / \mathrm{mim} / 1.73 \mathrm{~m}^{2}\right)=186 \times(\mathrm{Scr}) \\
\times(\text { Age }) \times(0.742 \quad \text { if female }) \quad \times \\
(1.210 \text { if black }) \quad\end{array}$ \\
\hline $\begin{array}{l}\text { Cockcroft- } \\
\text { Gault }\end{array}$ & $\begin{array}{l}\text { Ccr (ml/min }) \quad(140 \quad- \\
\text { Age } \times \text { Weight } \times(0.85 \text { if female }) \\
72 \times \text { Scr }\end{array}$ \\
\hline
\end{tabular}

Scr: serum creatinine, Ccr: creatinine clearance

\section{Proteinuria :-}

Normal urinary protein excretion in an adult is less than $100 \mathrm{mg} / 24 \mathrm{~h}$. Higher levels of excretion (more than $200 \mathrm{mg} / 24 \mathrm{~h}$ ) suggest that glomerular pathology allows the passage of macromolecules such/as albumin, which are not normally filtered. Excretion rates tend to increase on standing, during exertion, or with fever. Pressor agents such as angiotensin and norepinephrine tend to increase proteinuria.

In health, proteinuria results from tubular protein excretion, particularly Tamm-Horsfall protein. Albumin is the pre- dominant protein filtered by the glomerulus, and therefore it is the most consistent marker of glomerular pathology. In health, albumin contributes little to urinary proteinuria (around 12 $\mathrm{mg} / 24 \mathrm{~h}$ ), as proteins crossing the GBM are mainly reabsorbed and degraded via receptor-mediated endocytosis. This process shows a preference for cationic proteins and only a limited capacity for albumin, resulting in even minor glomerular abnormalities raising albuminuria. Microalbuminuria refers to albumin excretion in the range of 30 to 300 $\mathrm{mg} / 24 \mathrm{~h}$ ( 20 to $200 \mu \mathrm{g} / \mathrm{min}$ ). This equates to a urinary albumin-creatinine ratio (ACR) of 17 to $250 \mathrm{mg} / \mathrm{g}$ for menand 25 to $355 \mathrm{mg} / \mathrm{g}$ for women. not only defines the presence of CKD but also has important implications for diagnosis of the type of kidney disease and is associated with a worse prognosis for both kidney disease progression and the development of CVD (Sarnak et al., 2003).

Twenty-four hour or other timed collections were the traditional way to measure urine albumin excretion (UAE) but measuring urine albumin/creatinine ratio (ACR) in a spot collection of morning urine in the fasting state is currently recommended as a simple, quick and comparatively accurate way of determining albuminuria (Levey et al., 2003; American Diabetes Association, 2004; Busby and Bakris, 2004). Albuminuria is defined as an "ACR of $30 \mathrm{mg} / \mathrm{g}$ or higher", with microalbuminuria defined as an "ACR of 30 to $300 \mathrm{mg} / \mathrm{g}$ ", and macroalbuminuria defined as an "ACR over 300 mg/g" (Coresh et al., 2007).

\subsubsection{Classification of CKD}

Chronic kidney disease has been classified into various stages for the purpose of prevention, early identification of renal damage and institution of preventive measures for progression of the primary damage and appropriate guidelines for instituting management for prevention of complications in severe CKD (Vijayakumar et al., 2007).

National kidney foundation classified CKD into 5 stages according to the level of GFR (Table 2.3). For stages 1 and 2, kidney damage was assessed by spot albumin-to-creatinine ratio (NKF, 2002).

Table 4.3 Classification of the stages of chronic kidney disease

CKD GFRml/min /1.73 m2 Stages Description

1. Kidney damage with normal or increased GFR $\geq 90$

2. Kidney damage with mild reduction of GFR 60-89

3. Moderate reduction of GFR $30-50$

4. Severe reduction of GFR 15-29

5. Kidney failure $<15$ or dialysis

*Adopted from NKF, 2002.

\section{Cardiovascular disease in CKD patients:-}

Arterial vascular disease and cardiomyopathy are the primary types of CVD. In CKD, it is useful to consider two subtypes of arterial vascular disease, namely atherosclerosis and arteriosclerosis or large vessel remodelling (Sarnak et al., 2003). 
International Journal of Trend in Scientific Research and Development (IJTSRD) ISSN: 2456-6470

Table 4.4 Spectrum of cardiovascular disease in chronic

\begin{tabular}{|c|c|c|}
\hline $\begin{array}{l}\text { Types of } \\
\text { CVD }\end{array}$ & Pathology & \begin{tabular}{|c|} 
Clinical \\
presentations of \\
CVD
\end{tabular} \\
\hline $\begin{array}{l}\text { Arterial } \\
\text { vascular } \\
\text { disease }\end{array}$ & Atherosclerosis & $\begin{array}{l}\text { Atherosclerosis } \\
\text { IHD } \\
\text { (myocardial } \\
\text { infarction, } \\
\text { angina, sudden } \\
\text { cardiac death), } \\
\text { Cerebrovascular } \\
\text { disease, PVD, } \\
\text { HF }\end{array}$ \\
\hline & $\begin{array}{l}\text { Arteriosclerosis: } \\
\text { Dilated and non- } \\
\text { compliant large } \\
\text { vessels }\end{array}$ & $\mathrm{IHD}, \mathrm{HF}$ \\
\hline $\begin{array}{l}\text { Cardiomyo } \\
\text {-pathy }\end{array}$ & $\begin{array}{l}\text { Concentric } \\
\text { LVH, LV } \\
\text { dilatation with } \\
\text { proportional } \\
\text { hypertrophy }\end{array}$ & $\begin{array}{l}\mathrm{HF}, \\
\text { hypotension, } \\
\text { IHD }\end{array}$ \\
\hline
\end{tabular}

IMT: intima-media thickness, IHD: ischemic heart disease, HF: heart failure, LVH: left ventricular hypertrophy, PVD: peripheral vascular disease

Atherosclerosis is an intimal disease characterized by the presence of plaques and occlusive lesions in the wall of blood vessels (London et al., 2002). There is a high prevalence of atherosclerosis in CKD. Atherosclerotic lesions in CKD are frequently calcified, and have increased media thickness in comparison with lesions in the general population (Schwarz et al., 2000).

\subsubsection{Epidemiology of CVD in CKD:-}

Cardiovascular disease is frequently associated with CKD, and CKD appears to be a risk factor for CVD. The National Kidney Foundation, American Heart Association, and the Seventh Joint National Committee on Prevention, Detection, Evaluation, and Treatment of High Blood Pressure have classified the presence of chronic kidney disease as a cardiovascular risk factor (Shlipak et al., 2005).

National Kidney Foundation Task Force on Cardiovascular Disease in Chronic Renal Disease issued a report which showed that there was a high prevalence of CVD in CKD and that mortality due to CVD was 10 to 30 times higher in dialysis patients than in the general population. The task force recommended that patients with CKD be considered in the "highest risk group" for subsequent CVD events (Levey et al., 1998).

Cardiovascular disease accounts for approximately half of deaths in ESRD. The incidence of myocardial infarction and stroke in the dialysis population is 5 to 15 fold higher in ESRD. This increased risk is only partially explained by a high prevalence of atherosclerotic cardiovascular disease (ASCVD) and traditional ASCVD risk factors at the initiation of dialysis (Longenecker et al., 2002).

It was recognized that earlier stages of CKD also are a risk factor for CVD mortality. The risk for death from CVD was 1.8 times greater for persons with an estimated GFR of less than $70 \mathrm{~mL} / \mathrm{min}$ per $1.73 \mathrm{~m} 2$ than for those with a GFR of $90 \mathrm{~mL} / \mathrm{min}$ per $1.73 \mathrm{~m} 2$ or more after adjustment for age, race, sex, systolic blood pressure (SBP), serum total cholesterol level, BMI, diabetes, family history of CVD, physical inactivity, and level of education (Muntner et al., 2002). 


\section{Cardiovascular Disease in Patients with or Without CKD}

CKD: 2010

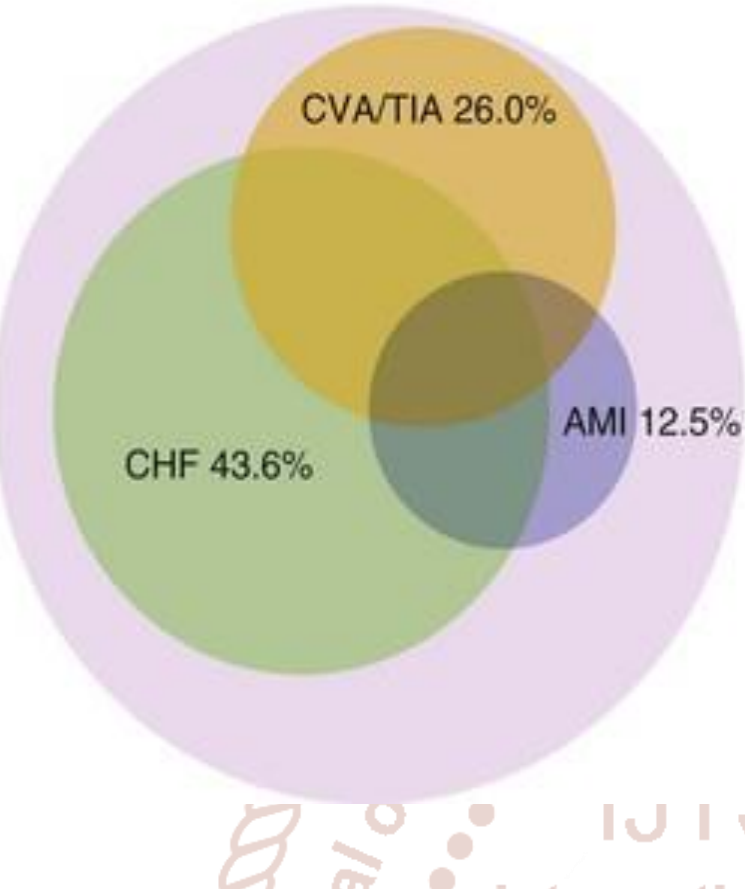

4.2.4 Cardiovascular disease risk factors in CKD

Cardiovascular disease risk factors are classified in CKD patients as either traditional or non-traditional (CKD specific) CVD risk factors. Traditional risk factors defined as those in the Framingham Heart Study that have been used to estimate the risk of developing symptomatic ischemic heart disease. Most of the traditional CVD risk factors are highly prevalent in CKD (Sarnak et al., 2003).
No CKD: 2010

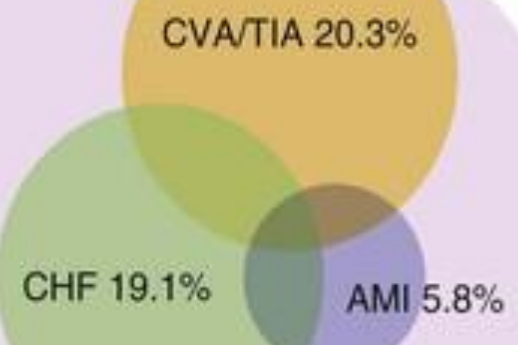

Table 4.5 Traditional and chronic kidney disease specific cardiovascular disease risk factors

\begin{tabular}{|l|l|}
\hline \multicolumn{1}{|c|}{ Traditional risk factors } & \multicolumn{1}{|c|}{ Nontraditional factors } \\
Older age & Albuminuria \\
Hypertension & Homocysteine \\
Higher LDL cholesterol & Lipoprotein(a) and apolipoprotein(a) \\
Lower HDL cholesterol & Isoforms \\
Diabetes & Lipoprotein remnants \\
Smoking & Anaemia \\
Physical inactivity Menopause & Abnormal calcium/phosphatemetabolism Extracellular fluid \\
Family history of CVD LVH & volume overload \\
& Electrolyte imbalance \\
& Oxidative stress \\
& Inflammation (C-reactive protein) \\
& Malnutrition \\
& Thrombogenic factors \\
& Sleep disturbances \\
& Altered nitric oxide/endothelia balance \\
\hline
\end{tabular}

LDL indicates low-density lipoprotein; HDL: high-density lipoprotein; LVH: left ventricular Hypertrophy. 


\section{Hypertension:-}

Definition:- Hypertension is defined as systolic blood pressure (SBP) of $140 \mathrm{mmHg}$ or greater, diastolic blood pressure (DBP) of $90 \mathrm{mmHg}$ or greater or greater or taking antihypertensive medication.

\begin{tabular}{|l|l|l|l|}
\hline S.NO. & \multicolumn{1}{|c|}{ Stage } & \multicolumn{1}{|c|}{$\begin{array}{c}\text { Systolic, } \\
\text { mmHg }\end{array}$} & $\begin{array}{c}\text { Diastolic, } \\
\text { mmHg }\end{array}$ \\
\hline 1. & Normal & $<120$ & $<80$ \\
\hline 2. & Prehypertension & $120-139$ & $80-89$ \\
\hline 3. & $\begin{array}{l}\text { Stage-1 } \\
\text { hypertension }\end{array}$ & $140-159$ & $90-99$ \\
\hline 4. & $\begin{array}{l}\text { Stage-2 } \\
\text { hypertension }\end{array}$ & $\geq 160$ & $\geq 100$ \\
\hline 5. & $\begin{array}{l}\text { Isolated systolic } \\
\text { hypertension }\end{array}$ & $\geq 140$ & $<90$ \\
\hline
\end{tabular}

Figure- showing Stages of hypertension

Hypertension is one of the most common traditional cardiovascular risk factors that is highly prevalent in CKD (Sarnak et al., 2003). Chronic kidney disease associated hypertension develops by a large variety of pathophysiological mechanisms. While fluid overload and activation of the renin-angiotensin system have long been recognized as crucial pathophysiological pathways, sympathetic hyperactivation, endothelial dysfunction and chronic hyperparathyroidism have more recently been identified as important factors contributing to CKD-associated hypertension (Hadtstein and Schaefer, 2008).
The prevalence of CKD-associated hypertension varies between $30 \%$ and $100 \%$ depending on the target population, cause of renal disease, and level of renal function (Blankestijn, 2005).

\section{Obesity and physical inactivity:-}

weight Body mass index (BMI) Body mass index is defined as a measurement of the relative percentages of fat and muscle mass in the human body, in which weight in kilograms is divided by height in squared meters and the result is used as an index of obesity (World Health Organization, WHO, 2000).

\section{Classification of BMI According to WHO:-}

\begin{tabular}{|cc|}
\hline Underweight & $<18.5 \mathrm{~kg} / \mathrm{m} 2$ \\
Normal weight & $18.5-24.9$ \\
$\mathrm{~kg} / \mathrm{m} 2$ & \\
Overweight $25-29.9 \mathrm{~kg} / \mathrm{m} 2$ \\
Obesity $\geq 30 \mathrm{~kg} / \mathrm{m} 2$ \\
Morbid obesity & $>40.0 \mathrm{~kg} / \mathrm{m} 2$ \\
\hline
\end{tabular}

People with inactive lifestyles or who have too much body fat have increased risk of CVD (Food and Drug Administration, 2004). Obesity and physical inactivity were associated significantly with CKD. Obesity may be the number one preventable risk factor for CKD due to its strong link with diabetes and hypertension, the two primary causes of CKD and kidney failure in adults (Kramer, 2006). 


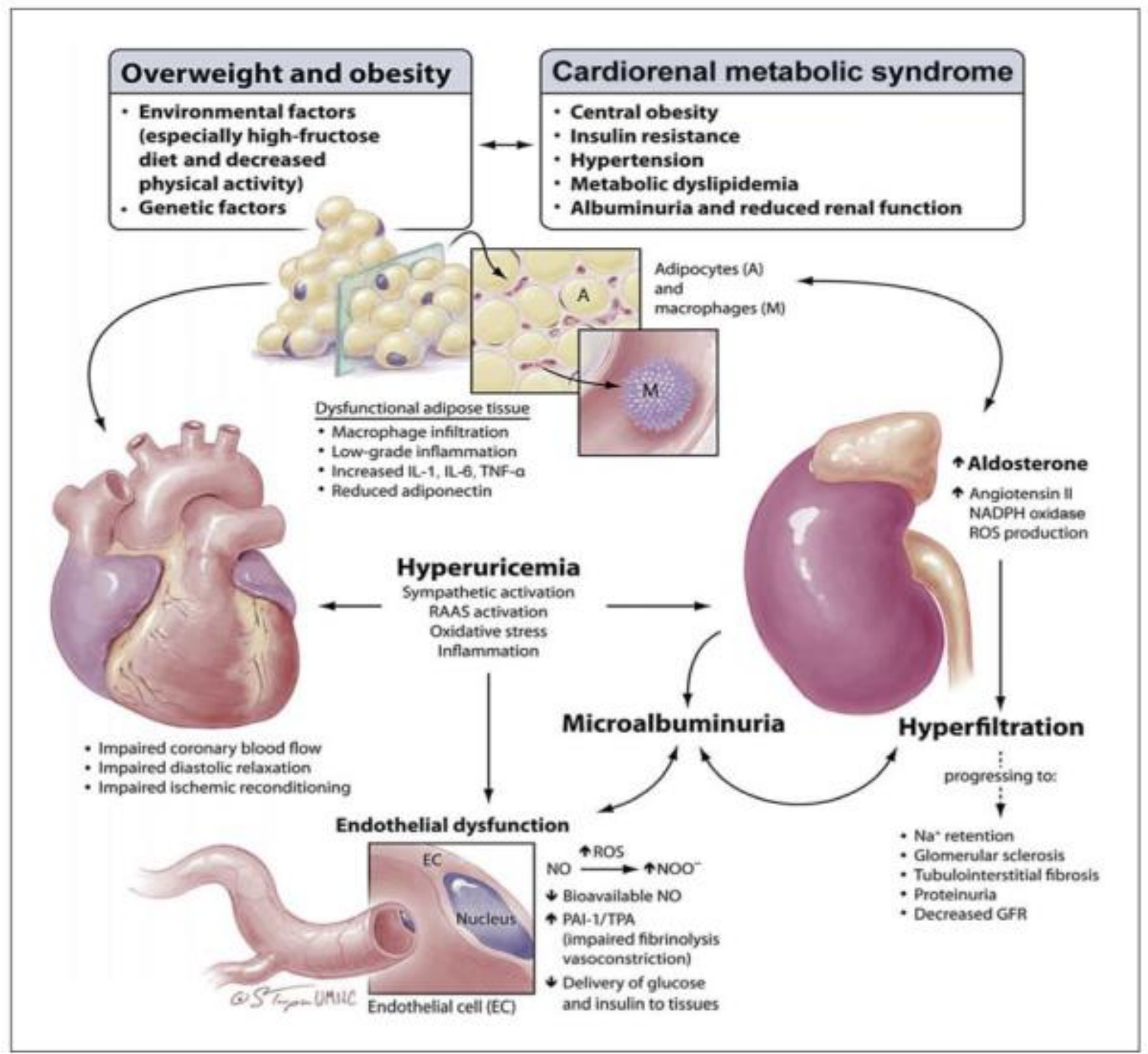

Figure showing:-cardiorenal metabolic syndrome

\section{Dyslipidemia :-}

Definition :-the American Heart Association states that lipidemia, generally referally referred to as hyperlipidemia is an hyperlipdimia, is an elevated concentration of lipids in the blood .elevated lipid ,or fat, in the blood produce high cholesterol and high triglycerides that contribute to heart disease.

\section{Reference range:-}

\begin{tabular}{|l|l|l|}
\hline Cholesterol & Critical value & $140-220 \mathrm{mg} / \mathrm{dl}$ \\
& risk High risk & $>220 \mathrm{mg} / \mathrm{dl}$ \\
\hline Triglycerides & High risk & $30-200 \mathrm{mg} / \mathrm{dl}$ \\
& & $>200 \mathrm{mg} / \mathrm{dl}$ \\
\hline
\end{tabular}

Dyslipidemia is any abnormality in plasma lipoprotein concentration or composition that is associated with an increased risk for atherosclerotic cardiovascular disease (ACVD) (NKF, 2003). Dyslipidemia has been established as a well-known traditional risk factor for CVD in the general population and large-scale observational studies have shown that total cholesterol and low density lipoprotein (LDL) values are two of the most important independent predictors of cardiovascular morbidity and mortality (Lewington et al., 2007).

Also, it is well-known that patients with impaired renal function exhibit significant alterations in lipoprotein metabolism. Although lipid abnormalities were originally considered as complications of ESRD, these changes can be present in early stages of CKD and may actively participate in the pathogenesis of serious complications such as ACVD (Tsimihodimos et al., 2008).

The most common quantitative lipid abnormalities in predialysis CKD patients are hypertriglyceridemia, increased concentrations of triglyceride-rich lipoprotein remnants, reduced high density lipoprotein (HDL) levels as well as increased concentrations of lipoprotein(Vaziri and Moradi , 2006). 


\section{Albuminuria:-}

Albuminuria is defined as $>30 \mathrm{mg} /$ day, if measured in a $24 \mathrm{~h}$ urine collection or over $30 \mathrm{mg} / \mathrm{g}$, if measured with the use of urinary ACR in a spot urine collection".

Urinary albumin excretion between 30 and 300 $\mathrm{mg} /$ day, or $30-300 \mathrm{mg} / \mathrm{g}$ is defined as microalbuminuria, any urinary albumin value below these limits is considered as normal UAE, whereas any value above them reflects the presence of macroalbuminuria or clinical proteinuria (American Diabetes Association, 2004; Kidney Disease Outcomes Quality Initiative, 2004).

It has long been noted that subclinical elevations of UAE are related to higher risk of subsequent development of clinical nephropathy in patients with diabetes mellitus, and that increased UAE was associated with higher risk for $\mathrm{CV}$ events and mortality in both diabetic and non-diabetic individuals (Mogensen, 1984; Stehouwer et al., 1992).

Prolonged protein loss leads to hypoalbuminemia, hyperlipidemia and coagulation //abnormalities following hyperfibrogenemia, increase in Factor III and Von Willebrand factor. Microalbuminuria occurring in early diabetic nephropathy is a marker of vascular endothelial dysfunction (Amaresan, 2005).

\section{Anaemia:-}

Anemia is simply a hemoglobin level lower than the normal range for a particular age and sex of the patient.

\section{The normal range for $\mathrm{Hb}$ and $\mathrm{RBC}$ :-}

$$
\begin{array}{llc}
\text { Males } & : 12-16 \mathrm{~g} / \mathrm{dL} & (4.0-5.5) \times 10 / \mathrm{L} \\
\text { Females }: 11-15 \mathrm{~g} / \mathrm{dL} & (3.5-5.0) \times 10^{12} / \mathrm{L} \\
\text { Neonates : } 17-20 \mathrm{~g} / \mathrm{dL} & (6.0-7.0) \times 10^{12} / \mathrm{L}
\end{array}
$$

Anaemia is defined as a low red blood cell count. Since the red blood cells contain haemoglobin, we simply measure its concentration. The kidney being the main source of erythropoietin, anaemia is apparently an integral part of advancing renal failure. Anaemia exerts an independent effect on CVD in CKD. For every $1 \mathrm{~g} / \mathrm{dl}$ drop in mean haemoglobin, the risk of cardiac failure increases by $25 \%$ and risk of death increases by $14 \%$ ( Metivier, 2000).

Depression of red cell production is the consequence of decreased renal production of the hormone erythropoietin. Increased red blood cell destruction resulting from uremic toxins as well as the mechanical damage to red cell observed in a variety of glomerular disease (Kissane, 1990)

\section{The pathophysiology of CVD induced by anaemia-}

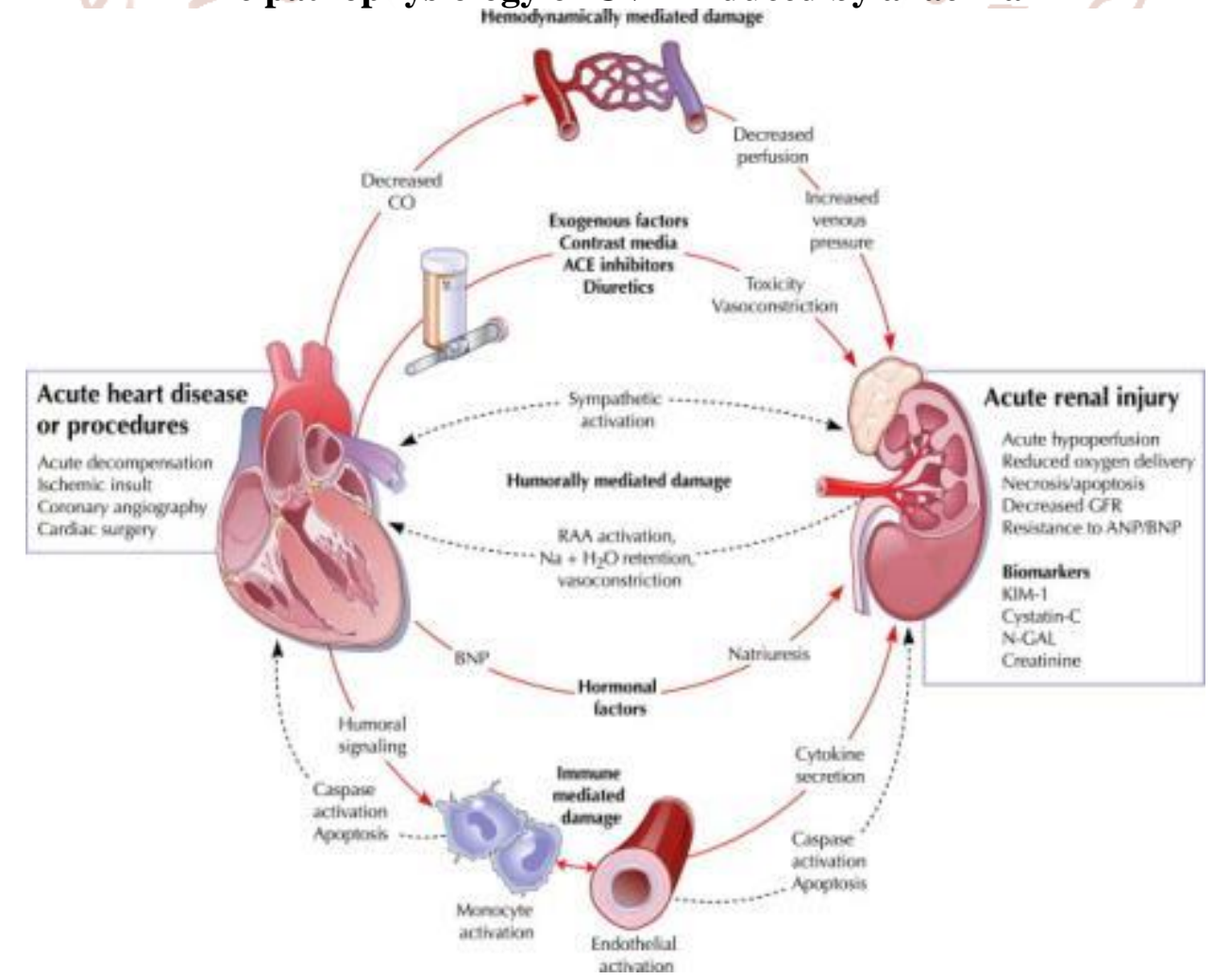

Figure 4.3. Adverse cardiovascular effects of anaemia 


\section{Calcium/phosphorus metabolism:-}

In humans with CKD, there appears to be a relationship between disorders of mineral metabolism (abnormal levels of serum calcium and phosphorus), abnormal bone (renal osteodystrophy), and vascular calcification (Moe and Chen, 2004).

Progressive nephron loss is associated with phosphate retention and hypocalcaemia early in the disease, but then can develop hypocalcaemia when given calcium or vitamin D. This triggers increased parathyroid hormone (PTH) activity. The secondary hyperparathyroidism along with hyperphosphatemia and increased calcium phosphate ion product are identified as independent cardiovascular risk factors (Brancaccio et al., 2002).

Secondary hyperparathyroidism results in impaired calcitrol synthesis, increased skeletal resistance to PTH, increased parathyroid cell hyperplasia, and decreased expression of calcium sensing receptors. These end in decreased cardiac contraction, LVH and valvular calcification.

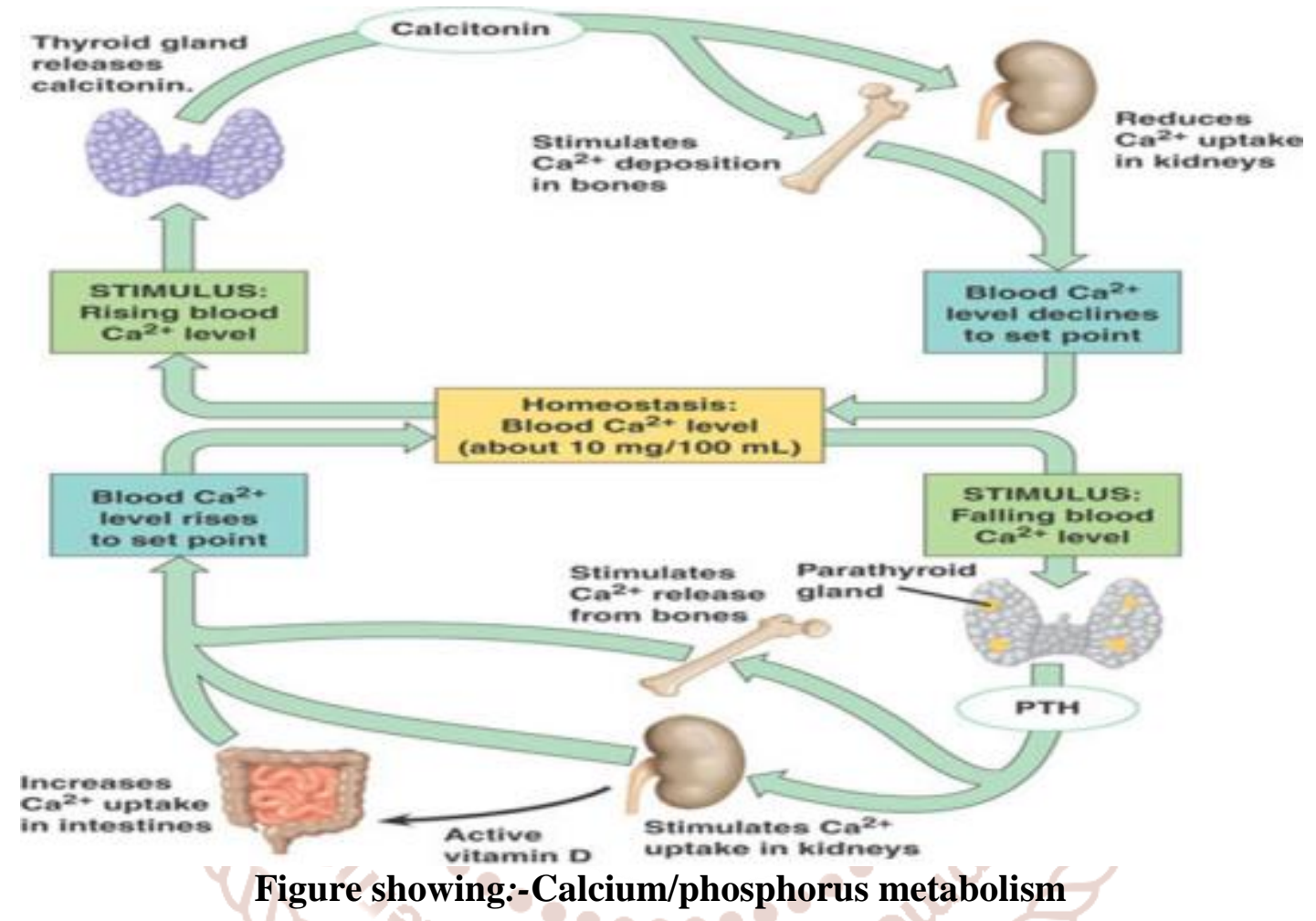

\section{Observation}

\subsection{Characteristics of the study population}

The present study is a cross sectional study, that included 50 patient of CKD among haemodialysis.

\subsubsection{Socio-demographic characteristics of the study population}

The distribution of the study population by gender showed that males represented $34(68 \%)$ and of the females represented $16(32 \%)$ of the patients.

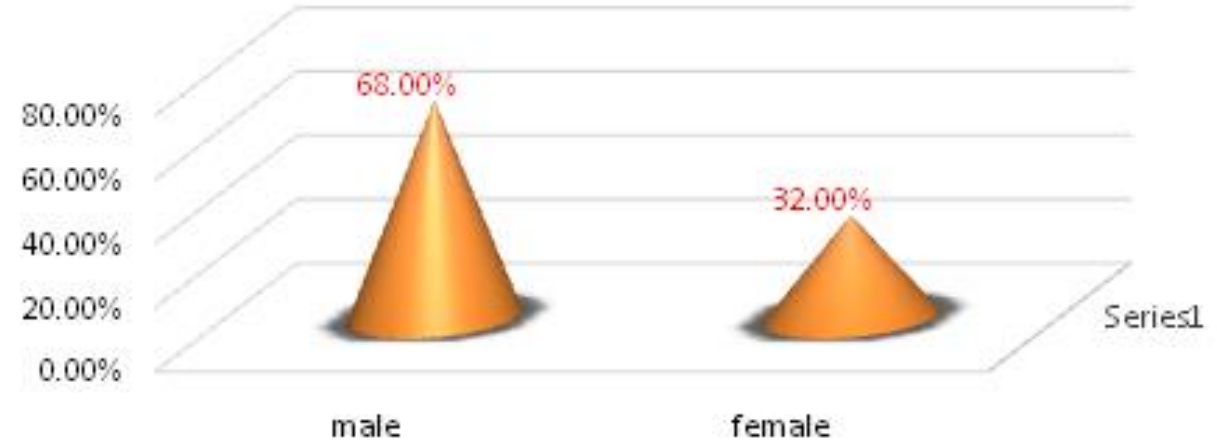

Figure 5.1.1: showing male/female percentage in CKD patient on haemodialysis 


\subsubsection{Epidemiological dada:-}

\begin{tabular}{|l|l|l|l|}
\hline Age(year) & \multicolumn{1}{|c|}{ Male \% } & Female \% & $\begin{array}{l}\text { Total no of } \\
\text { patient \% }\end{array}$ \\
\hline $10-20$ & $1(3.1 \%)$ & $0(0 \%)$ & $1(2 \%)$ \\
\hline $30-40$ & $12(37.5 \%)$ & $7(38.8 \%)$ & $19(38 \%)$ \\
\hline $50-60$ & $15(46 \%)$ & $11(61.1 \%)$ & $26(52 \%)$ \\
\hline $70-80$ & $3(9.3 \%)$ & $0(0 \%)$ & $3(6 \%)$ \\
\hline$>80$ & $1(3.1 \%)$ & $0(0 \%)$ & $1(2 \%)$ \\
\hline total & $\mathbf{3 2}(\mathbf{1 0 0 \%})$ & $\mathbf{1 8}(\mathbf{1 0 0 \%})$ & $\mathbf{5 0}(\mathbf{1 0 0 \%})$ \\
\hline
\end{tabular}

5.1.3Etiology factor of CVD in CKD patient on haemodia
\begin{tabular}{|l|l|l|}
\hline S.n. & \multicolumn{1}{|c|}{ Etiology } & $\%$ \\
\hline 1. & Hypertension & $37 \%$ \\
\hline 2. & Chronic glomerulonephritis & $8.6 \%$ \\
\hline 3. & Diabetes & $21 \%$ \\
\hline 4. & Diabetes with HTN & $10 \%$ \\
\hline 5. & PKD & $7.1 \%$ \\
\hline 6. & Analgesic nephropathy & $7.1 \%$ \\
\hline 7. & Other & 4.31 \\
\hline 8. & Obstructive uropathy & $5.7 \%$ \\
\hline
\end{tabular}

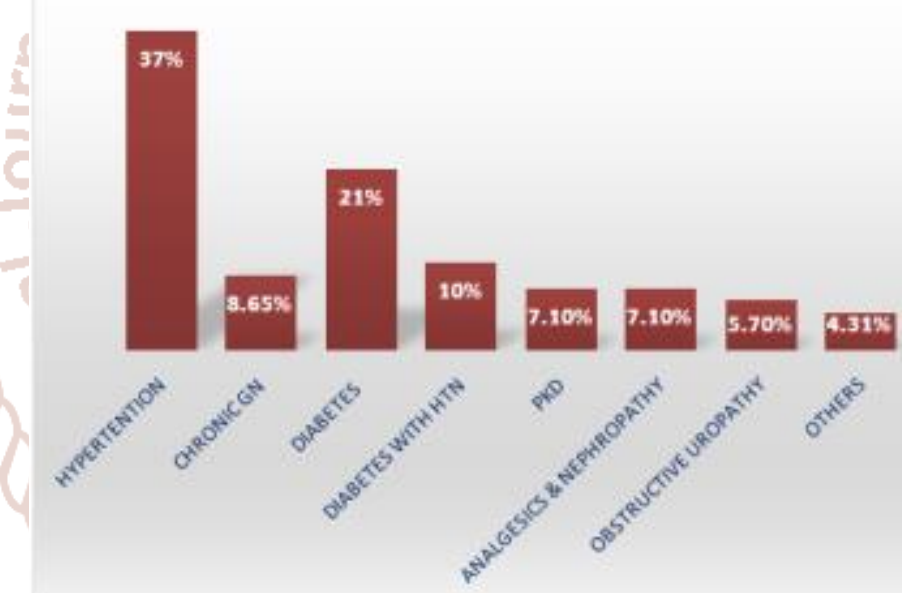

\section{Graph showing:- Etiology factor of CKD patient}

Table 5.1.3 shows the mean values of, weight, systolic contrast there were significant increases in mean of and diastolic blood pressure in patients and controls. the systolic pressure $(148.163 \pm 21.6345 \mathrm{mmHg})$ and There were significant decreases in mean values of diastolic blood pressure in CKD patients is weight of patients as compare $(54.20+-8.5341 \mathrm{Kg})$. In $\quad(82.8 \pm 7.4998 \mathrm{mmHg})$

Table 5.1.3 Means of weight, systolic and diastolic blood pressure in patients

\begin{tabular}{|l|l|}
\hline Variable & \multicolumn{1}{|c|}{ Patients $(\mathrm{n}=50)$ Mean \pm SD } \\
\hline Weight $(\mathbf{k g})$ & $54.20+8.5341$ \\
\hline SBP $(\mathbf{m m H g})$ & $148.163 \pm 21.6345$ \\
\hline DBP $(\mathbf{m m H g})$ & $82.8 \pm 7.4998$ \\
\hline
\end{tabular}


Graph showing echo finding in 50 patent of ckd pt among HD Concentric LVH ( $\mathrm{n}=56 \%$ ),LVDD $(n=20 \%)$,puller effusion $(n=16 \%)$ and control group under $(n=8 \%)$

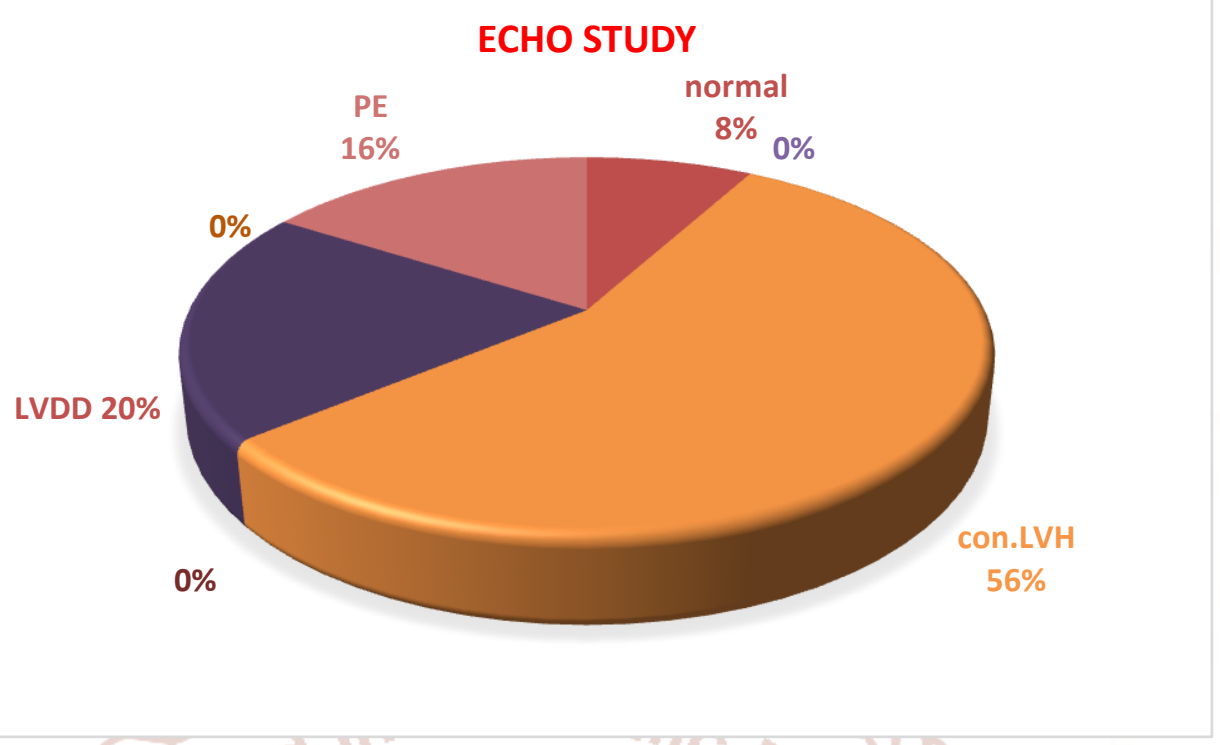

Graph:- showing Echo finding in CKD patients among haemodialysis

Our 50 patient study the ECG finding showing normal $20 \%$ patients, sinus tachycardia $52 \%$ patients, tall Twave $30 \%$ patients, and poor $\mathrm{R}$-wave $8 \%$ patients.

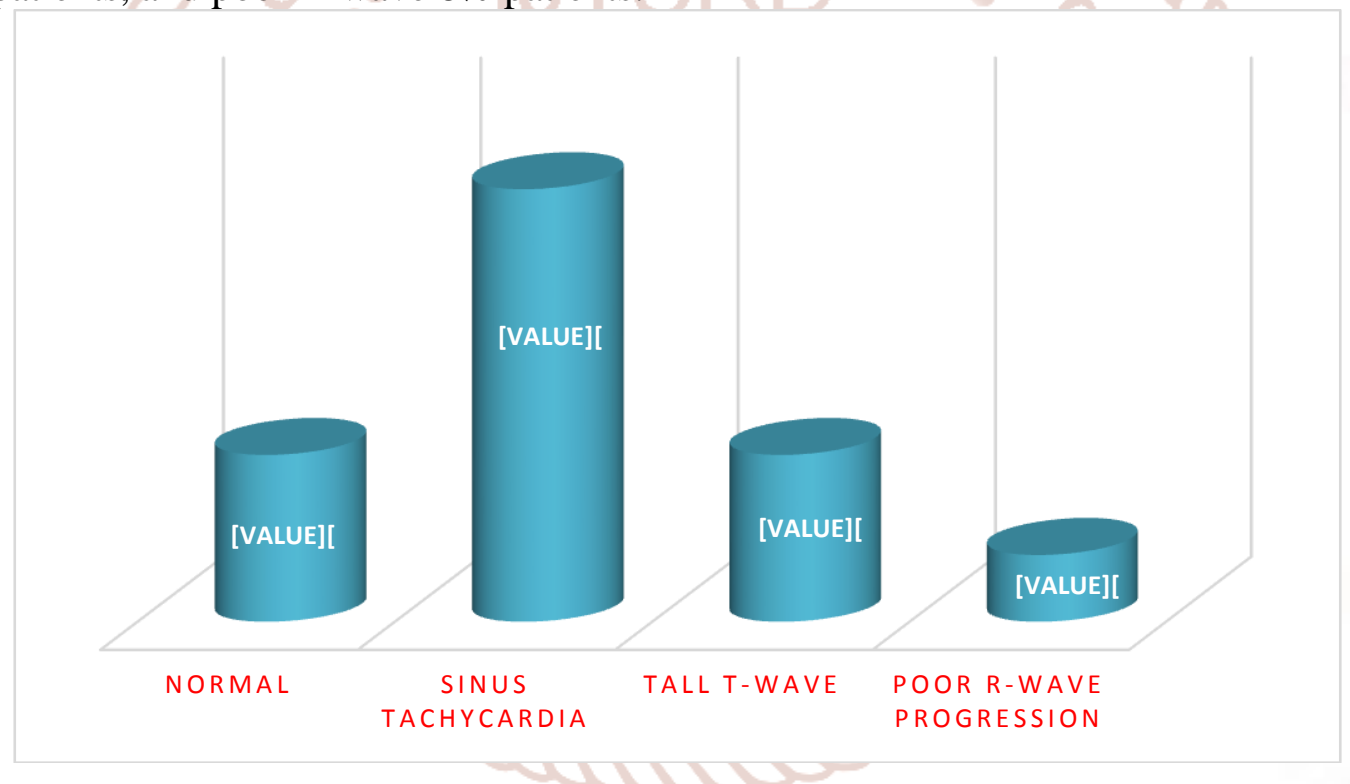

Figure:-showing ECG finding among hemodialysis patient

\subsubsection{Kidney investigations and CKD classification}

All the participants were tested for the function of the kidney. Glomerular filtration rate was determined from serum creatinine and subjects height according to Schwartz formula . Albumin/creatinine ratio was determined from spot urine sample to indicate the damage of the kidney and used as a marker of CVD event. Glomerular filtration rate and ACR were used in defining and classifying CKD.
Table 5.1.4 illustrates the comparison of the mean values for urea, creatinine, ACR and GFR between patient.

The results showed significant patients in the mean values of urea $(172.44+-68.6184 \mathrm{mg} / \mathrm{dl})$. The mean values of creatinine $(10.304 \pm 3.63 \mathrm{mg} / \mathrm{dl})$ with statistically .the mean values of ACR $(0.63755 \pm 0.34833)$ and mean values of $\operatorname{GFR}(5.6 \pm 2.615339)$ 
International Journal of Trend in Scientific Research and Development (IJTSRD) ISSN: 2456-6470

Table 5.1.4 investigations of CKD patients on haemodialysis

\begin{tabular}{|l|l|}
\hline \multicolumn{1}{|c|}{ Kidney function tests } & \multicolumn{1}{c|}{ Mean \pm SD } \\
\hline Urea $(\mathrm{mg} / \mathrm{dl}) \quad(\mathrm{n}=50)$ & $172.44+-68.6184$ \\
\hline Creatinine $(\mathrm{mg} / \mathrm{dl})(\mathrm{n}=50)$ & $10.304 \pm 3.63$ \\
\hline ACR $(\mathrm{mg} / \mathrm{g}) \quad(\mathrm{n}=50)$ & $0.63755 \pm 0.34833$ \\
\hline GFR $(\mathrm{ml} / \mathrm{min} / 1.73 \mathrm{~m} 2)(\mathrm{n}=50)$ & $5.6 \pm 2.615339$ \\
\hline
\end{tabular}

The patients were classified into 1 to 5 stages according to the NKF- KDOQI (Table 5.3). Figure 4.5 shows the percent distribution of the patients in each stage of CKD. The highest number of the patients belonged to stage 5 (49 patients, ) followed by stage 4 (1 patients,), stage 3 (0patients), and then stage 2(0 patients,). Stage1 (0 patient).

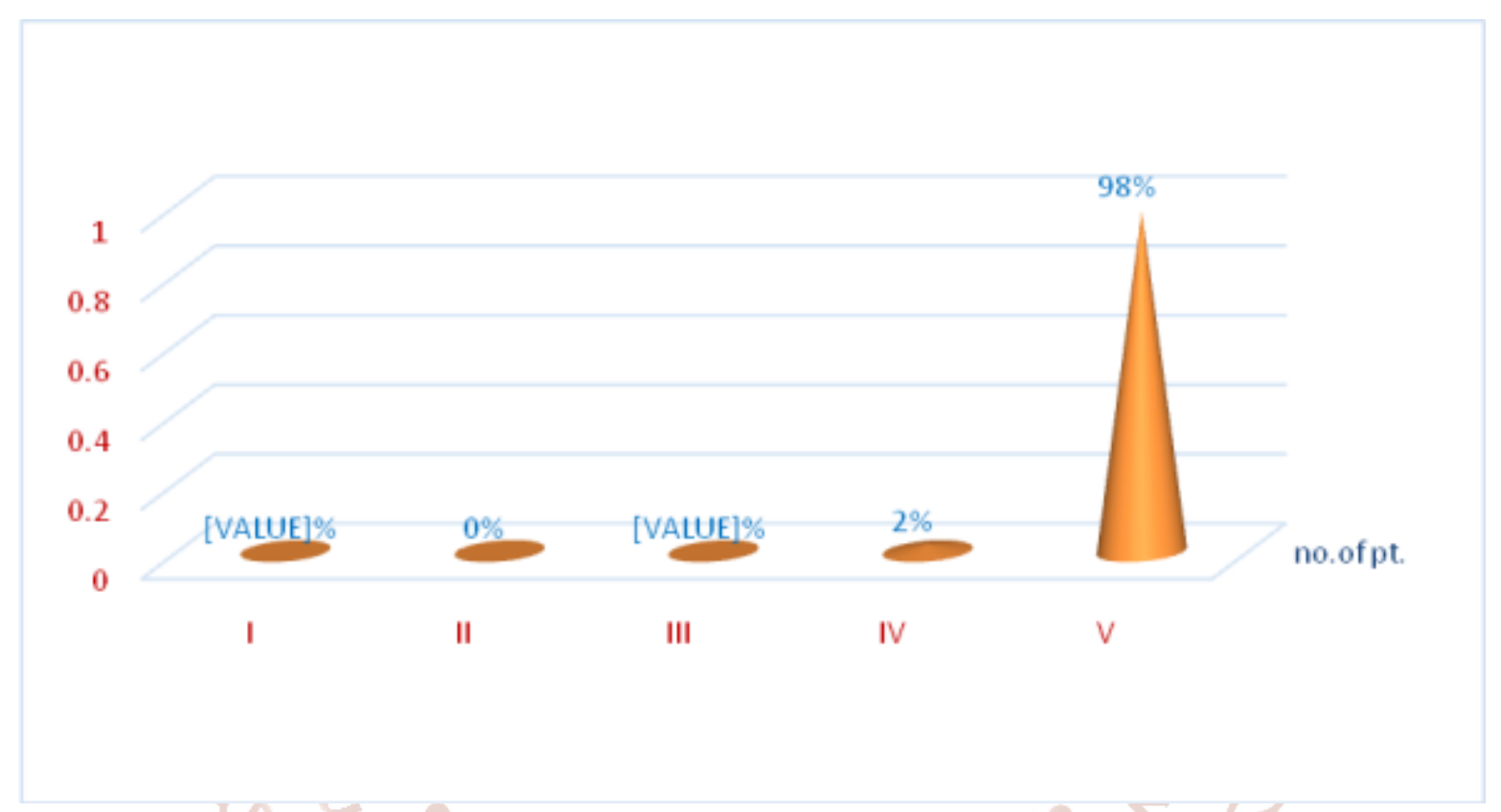

Figure 5.1.5 Distribution of chronic kidney disease stages among haemodialysis patients

VARIATION OF FREQUENCY OF CARDIAC DISEAES ACROSS THE DIFFERENT STAGES:-

IN ECHO STUDY:-

\begin{tabular}{|l|l|l|l|l|l|}
\hline VARIABLE & $\begin{array}{c}\text { STAGE 1 } \\
\text { no } \\
\text { application }\end{array}$ & $\begin{array}{c}\text { STAGE 2 } \\
\text { No } \\
\text { applicable }\end{array}$ & $\begin{array}{c}\text { STAGE 3 } \\
\text { No } \\
\text { applicable }\end{array}$ & \multicolumn{1}{|c|}{$\begin{array}{c}\text { STAGE } \\
\mathbf{4}(\mathbf{n = 2})\end{array}$} & $\begin{array}{c}\text { STAGE } \\
\mathbf{5}(\mathbf{n}=\mathbf{4 8})\end{array}$ \\
\hline LVH n(\%) & - & - & - & $10 \%$ & $56 \%$ \\
\hline LVDD n(\%) & - & - & - & $8 \%$ & $20 \%$ \\
\hline PE n(\%) & - & - & - & $7 \%$ & $16 \%$ \\
\hline LVSF n(\%) & - & - & - & $4 \%$ & $7 \%$ \\
\hline IHD n(\%) & - & - & - & $0 \%$ & $0 \%$ \\
\hline EFn (<50\%) & - & - & - & $4 \%$ & $23 \%$ \\
\hline $\begin{array}{l}\text { NORMAL } \\
\mathrm{n}(\%)\end{array}$ & - & - & - & $12 \%$ & $8 \%$ \\
\hline
\end{tabular}


ECG FINDINGS:-

\begin{tabular}{|l|l|l|l|l|l|}
\hline VARIABLE & $\begin{array}{l}\text { STAGE 1 no } \\
\text { applicable }\end{array}$ & $\begin{array}{l}\text { STAGE 2 no } \\
\text { applicable }\end{array}$ & $\begin{array}{l}\text { STAGE 3 no } \\
\text { applicable }\end{array}$ & $\begin{array}{l}\text { STAGE } \\
4(\mathrm{n}=2)\end{array}$ & $\begin{array}{l}\text { STAGE 5 } \\
(\mathrm{n}=48)\end{array}$ \\
\hline LVH & - & - & - & $30 \%$ & $56 \%$ \\
\hline $\begin{array}{l}\text { SINUS } \\
\text { TACHYCARDIA }\end{array}$ & - & - & - & $37 \%$ & $52 \%$ \\
\hline TALL T-WAVE & - & - & - & $12 \%$ & $20 \%$ \\
\hline $\begin{array}{l}\text { POOR R - } \\
\text { WAVE } \\
\text { PROGRESSION }\end{array}$ & - & - & - & $8 \%$ & $10 \%$ \\
\hline NORMAL & - & - & - & $27 \%$ & $20 \%$ \\
\hline
\end{tabular}

Table 5.1.6 shows the mean values of cholesterol, triglycerides,

the patients showed significantly mean values of cholesterol $(145.08 \pm 45.3538 \mathrm{mg} / \mathrm{dl})$ and mean values of triglyceride $(146.102 \pm 85.6922 \mathrm{mg} / \mathrm{dl}$, )and mean values of HDL $36.34 \pm 19.6495 \mathrm{mg} / \mathrm{dl}$

\begin{tabular}{|l|l|}
\hline \multicolumn{1}{|c|}{ Lipid profile } & \multicolumn{1}{|c|}{ Patient $(\mathrm{n}=50)$} \\
\hline Cholesterol (mg/dl) & $145.08 \pm 45.3538$ \\
Triglycerides (mg/dl) & $146.102 \pm 85.6922$ \\
HDL (mg/dl) & $36.34 \pm 19.64954$ \\
\hline
\end{tabular}

The graph show 50 patient study population, cholesterol, normal $(n=39)$ high concentrations of cholesterol( $(n=7)$ and low cholesterol $(n=4)$. Our study showed significant increase in total cholesterol, triglycerides and LDL in the patients as compared to the controls. The results also revealed significantly lower level of HDL in the patients.

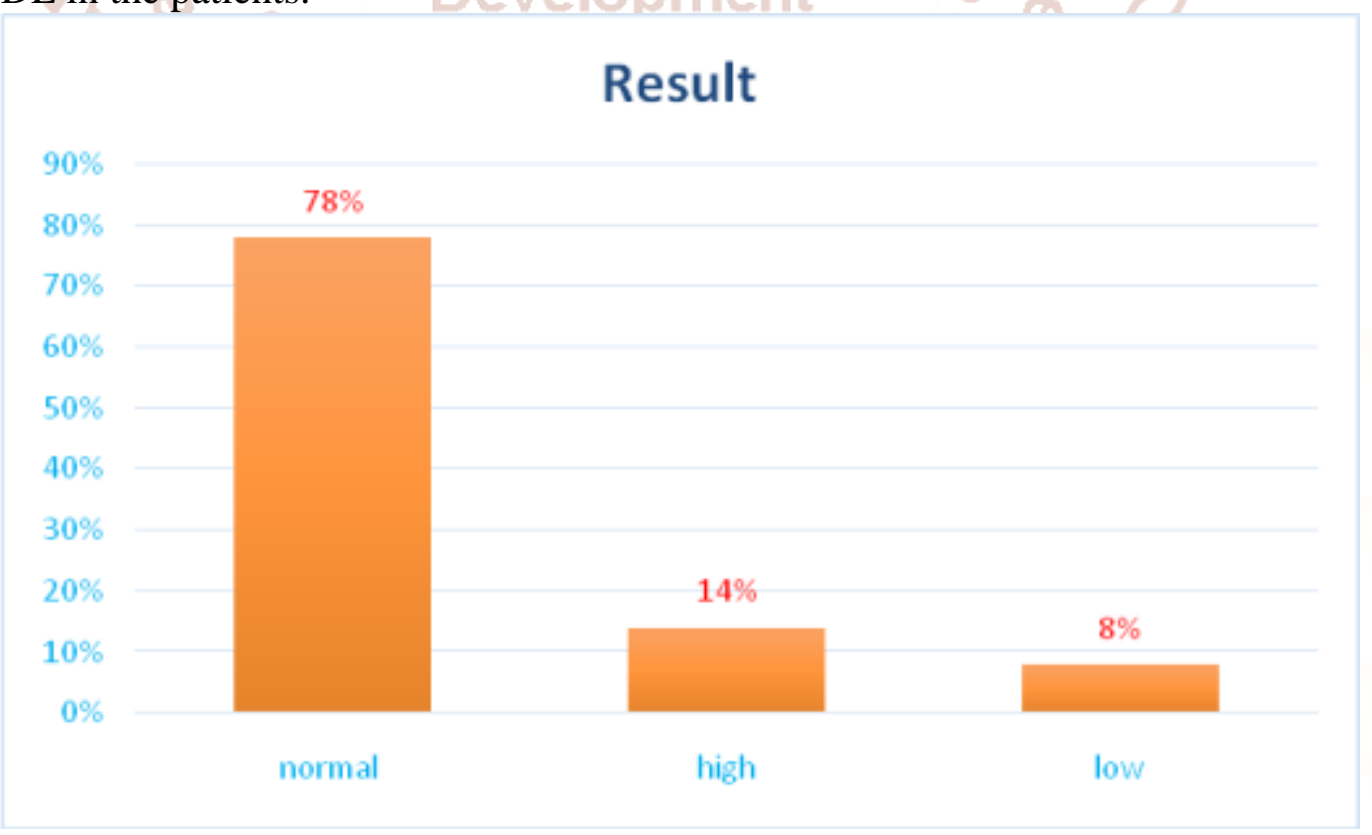

Graph: showing Cholesterol among dialysis patient

There seems to be a gradual shift to the uremic lipid profile as kidney function deteriorates . Uremic lipid profile shows dyslipidaemia with elevated triglyceride and decreased HDL. normal triglyceride $(n=36)$, High triglyceride $(\mathrm{n}=13$ ) and low triglyceride $(\mathrm{n}=1)$ 




\section{Graph: showing Triglyceride among dialysis patient}

\subsubsection{Chronic kidney disease- specific CVD risk factors}

The participants were investigated for some of CVD risk factors that are considered specific to CKD such as anaemia, and albuminuria.

Table 5.1.8 shows that $50 \mathrm{pt}$. study most of the patients were anaemic $(\mathrm{n}=48)$, while normal in the control group $(\mathrm{n}=2)$ And 43 patient is protineuria and 7 patient is normal.

\begin{tabular}{|l|l|l|}
\hline CKD-specific CVD risk factors & \multicolumn{1}{|c|}{ Patient $(\mathrm{n}=\mathbf{5 0})$} & \multicolumn{1}{|c|}{ Control group } \\
\hline Anaemia & 48 & 2 \\
Albuminuria & $\begin{array}{l}48 \\
43\end{array}$ & 7 \\
\hline
\end{tabular}

\subsection{8albuminuria}

graph 4.9 the normal and proteinuria between patients. The results indicated that there was a significant difference between the means of the patients and mean of haemoglobin $(9.51 \pm 1.156 \mathrm{~g} / \mathrm{dl})$, ACR $(0.6375 \pm 0.348334 \mathrm{gm})$, albuminuria, the majority of the patients (43) had albuminuria and normal 7 patient.

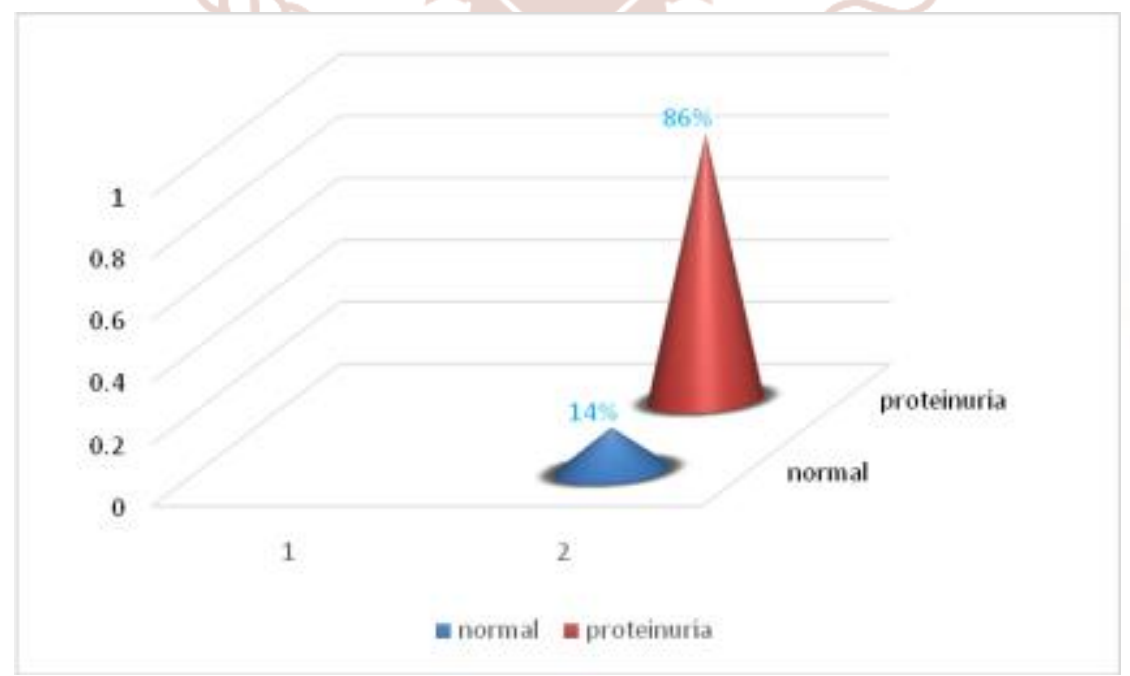

Graph: - showing proteinuria and normal condition in CKD pt among HD 
Table 5.1.9 Means of some chronic kidney disease- specific cardiovascular disease risk factors in CKD patients

\begin{tabular}{|ll|l|}
\hline \multicolumn{2}{|c|}{ CKD-specific CVD risk factors } & \multicolumn{1}{c|}{ Mean \pm SD } \\
\hline Haemoglobin $(\mathrm{g} / \mathrm{dl})$ & patient $(\mathrm{n}=50)$ & $9.51 \pm 1.156$ \\
& & $0.6375 \pm 0.34833$ \\
ACR $(\mathrm{gm})$. & patient $(\mathrm{n}=50)$ &
\end{tabular}

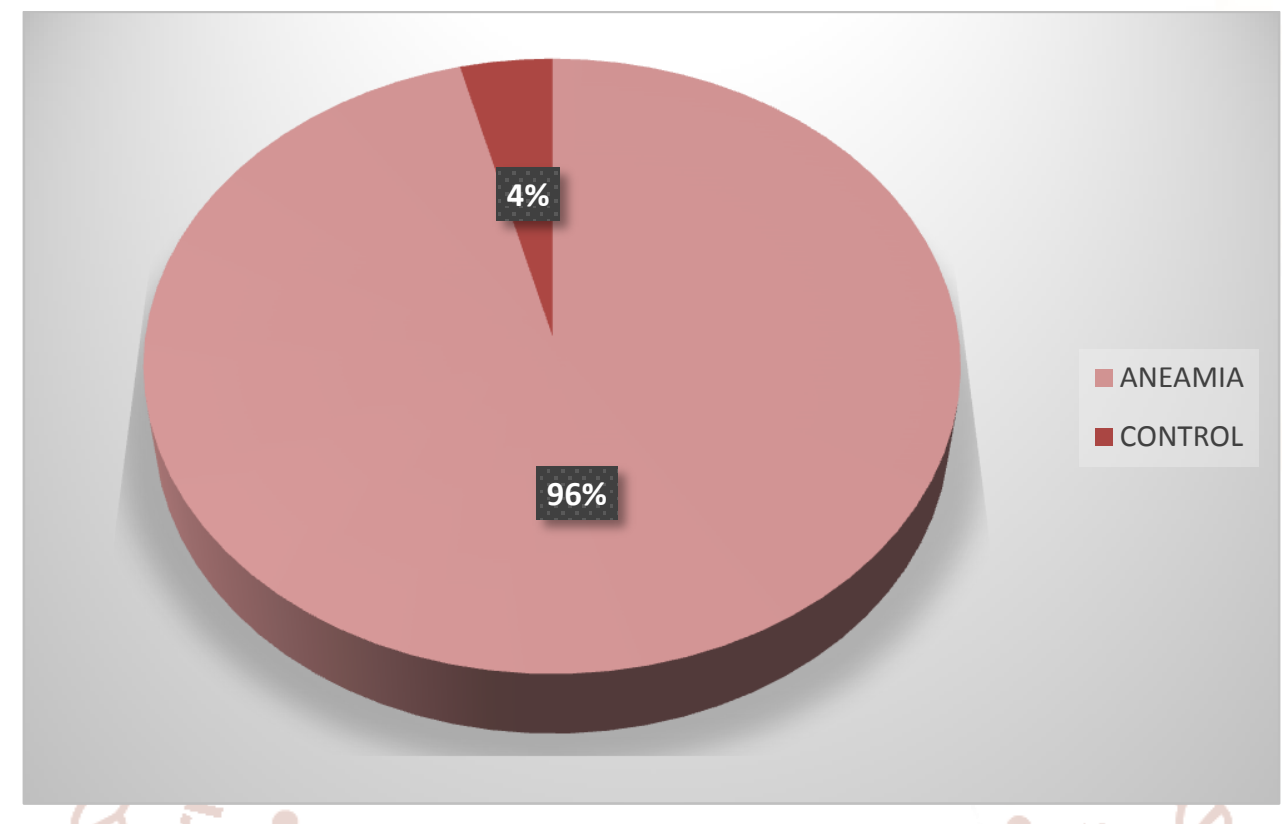

Graph- showing anaemia $96 \%$ and control group $4 \%$ among haemodialysis patient.

\section{Discussion}

Cardiovascular disease is frequently associated with CKD. Cardiac problems are more clear in ESRD and dialysis patients, some of the CVD risk factors are frequent and appear early in CKD. Detection and treatment of CVD risk factors is important to control the progression of the disease, since some of these risk factors are treatable and controllable.

\subsection{Characteristics of the study population}

\subsubsection{Socio-demographic characteristics of the} study population

The present study was conducted on 50 dialysis CKD patients aged $>17$ to 83 years old in the Nephrology Department Dr B. R. A. M.hospital RAIPUR C.G. Healthy control group was also included in the study. The study showed that the male sex $(68.00 \%)$ was higher than female $(32.00 \%)$. Age group 17-83 years was more frequent the progressive nature of the disease. Population density and presence of Dr. B. R. A. M. Hospital Raipur may be the cause of the higher number of subjects. Educational level of participants revealed high distribution of illiterate.

\subsection{2 weight, pulse, systolic and diastolic blood} pressure among patients and controls

The study showed statistically significant differences between patients and controls with respect to height, weight, pulse rate, systolic and diastolic blood pressure, where the mean values indicated that the patients were lower weight, higher systolic and diastolic blood pressure.

This result is congruent to that of Litwin et al. (2005) who found that the patients were significantly higher blood pressure than control subjects. Other studies have indicated that CKD in growing adult leads to a state of impaired growth due to altered metabolic status and defective growth hormone action (Muthukrishnan et al., 2007), and that high blood pressure is a leading cause of CKD in adults. High blood pressure can also be a complication of kidney disease (NKF, 2009)

\section{Stages of CKD in the patients:-}

Patients of the study were classified into 1 to 5 stages as recommended by NKF-KDOQI.; the 1 cases found and 49 patient under stage 5 of CKD. no -7 patients 
had normal ACR while other patients had abnormal ACR 43 albuminuria. The results showed that the patients enrolled in this study could be classified into stages of CKD stage 1, 2, 3 where stage was not present. Stage 4 rare incidence, followed by stage 5 , The increased presentation of stage 5 in the study population is due to initiation of haemodialysis dialysis for many of stage 5 patients and in some cases death of the patients before reaching stage 5 . Absence of stage 1,2 ,3,these stages referred and hospitalized in the Nephrology Department of DR. B.R.A.H. RAIPUR. since the disease in such early stages is asymptomatic and criteria of kidney impairment is very mild especially in stage 1 . Unfortunately no data regarding the prevalence of CKD and CKD stages is available in our populations to compare the study findings.

\subsection{Distribution of traditional CVD risk factors in study sample}

\subsubsection{Hypertension}

The present study showed that there is association between hypertension and CKD. Forty seven percent of patients had a history of hypertension and were given the anti-hypertensive drugs. The means of systolic and diastolic blood pressure were significantly higher in patients as compared to controls. The relationship between hypertension and progression of CKD was clear within stages of CKD since the frequency of hypertensive patients gradually increased through stages 2,3 and 5.

The results are in agreement with those of other authors who found an association between hypertension and CKD and showed that hypertension was frequent in all stages of CKD (Goicoechea et al., 2005; Zhang et al., 2006; Parikh et al., 2006.

Database estimated the prevalence of hypertension among Adult with CKD as being closer to $50 \%$ and demonstrated that renal function in hypertensive adult with CKD deteriorated significantly more rapidly than in normotensive adult, and raises the possibility that improved blood pressure control may be one method of slowing the progression of CKD in this population (Mitsnefes et al., 2003).

In another study that was carried out on CKD adult patients not receiving renal replacement therapy showed that $76 \%$ of patients had history of hypertension. Locatelli et al. (2003) found out that hypertension plays a major role in determining cardiac damage at all stages of $\mathrm{CKD}$, including the dialytic phase.(Wheeler et al., 2003)

\subsubsection{Physical inactivity, obesity and family history of CVD}

Half of the patients of the present study were physically inactive. Frequency of physical inactivity among patients was two-fold higher as compared to controls. With respect to $\mathrm{CKD}$ stages the results showed that all cases in stage 2 were active, inactivity was increased in stages 3 and 4, and in stage 5 all cases were inactive. This indicates a positive correlation between physical inactivity and CKD progression. (2005) stated that low physical activity is a predictor of cardiovascular mortality in persons with chronic kidney disease. In the present study BMI was used as an indicator for obesity.

The results showed that the frequency of underweight among patients was higher than controls. Overweight and obesity were not encountered in the study population. Chronic kidney disease stages in the study revealed that the frequency of underweight increased as GFR declined through stages 2, 4 and 5. Our results are in accordance with the study of Litwin et al. (2005) who showed that patients with CKD were lighter and had lower BMI than controls. The variation in the results may be because we studied the presence of obesity in our study population who are already suffering from CKD and not CKD as a sequel to obesity. In studying family history of CVD as a risk factor of CVD in the study population, the results indicated that there was no difference between patients and controls since they had nearly equal percentages of subjects with family history of CVD. This observation indicates that there is a genetic factor in causing CVD.

\subsubsection{Dyslipidaemia}

In the general population, high plasma concentrations of LDL cholesterol, low concentrations of HDL cholesterol, and to some extent high total triglyceride concentrations are associated with increased atherosclerotic CVD risk (Wilson et al., 1998). There seems to be a gradual shift to the uremic lipid profile as kidney function deteriorates (Kronenberg et al., 2002).

Uremic lipid profile shows dyslipidaemia with elevated cholesterol, triglyceride and decreased HDL. Elevated plasma LDL cholesterol concentration is common in nephrotic syndrome but is not a typical feature of patients with advanced CKD 
(Bonnie et al., 2007).

Our study showed significant increase in total cholesterol, triglycerides and LDL in the patients as compared to the controls. The results also revealed significantly lower level of HDL in the patients. A gradual shift to the uremic lipid profile as CKD progresses was evident in CKD stages of the present study (gradual increase of cholesterol, triglycerides, LDL, and decrease in the levels of HDL). This finding is in agreement with other studies that assessed the association between CKD and some CVD risk factors including dyslipidemia. Studies conducted on older patients in different stages of CKD found that the CKD patients had high prevalence of dyslipidemia (Locatelli et al., 2003; Zhang et al., 2006; Shroff, 2009). Wheeler et al. (2003) also showed that there is an association between CKD and dyslipidemia with reduced LDL, elevated triglyceride and decreased HDL levels. the control group and patient groups were comparable in terms of dyslipidemia, the patients had significantly higher triglycerides levels than controls, whereas total cholesterol and LDL were selectively increased in the dialysis group (Litwin et al., 2005).

\subsection{Chronic kidney disease specific CVD risk factors:-}

\subsubsection{Albuminuria}

Elevation of albuminuria is related to higher risk of subsequent development of clinical nephropathy and that increased albuminuria is associated with higher risk for CVD events (Mogensen, 1984).

More than one method can be used to determine urine albumin execration (KDOQI, 2004). In the present study ACR in spot morning urine samples was used to measure albuminuria.

The results showed that there was association between CKD and increased level of ACR with significant difference between patients and controls. Macroalbuminuria (ACR> $300 \mathrm{mg} / \mathrm{g}$ ) was predominant in the patients followed by microalbuminuria $(\mathrm{ACR}=30-300 \mathrm{mg} / \mathrm{g})$, while few patients $(8.3 \%)$ had normal albuminuria $(\mathrm{ACR}<30$ $\mathrm{mg} / \mathrm{g}$ ). Albuminuria showed irregularity between stages of CKD, i.e. ACR levels fluctuated between stages.

This irregularity can be attributed to the cause of the renal disease, since albuminuria varies according to the cause of the renal disease (Sarnak et al., 2003).
Our findings are congruent with other studies, which suggested that albuminuria is a common feature in $\mathrm{CKD}$ and is related to the underlying glomerular or interstitial damage reported that albuminuria was frequent even in early stages of CKD.

\subsubsection{Anaemia}

Anaemia has been shown to be significantly associated with $\mathrm{LVH}$ in both dialysis patients and patients with early CKD, suggesting that anaemia is primarily implicated in the development of $\mathrm{LVH}$ in CKD (Silverberg et al., 1998 and Levin et al., 1999).

Anaemia is a potentially modifiable risk factor; its treatment seems to have a more beneficial effect on $\mathrm{LVH}$ regression if it is given as early as possible during the course of CKD and before patients have reached ESRD

In the present study, the majority of patients were anaemic and the mean values of haemoglobin 9.51 showed significant decrease in patients as compared to controls. The results showed continuous decrease of haemoglobin in stages 3, 4 and 5, which indicate an association between anaemia and CKD progression in the study population. (Locatelli et al., 2003)

\section{CONCLUSION\& RECOMMENDATION}

Our patients study in 50 patient of CKD among hemodialysis, there were 34 males $(68 \%)$ and 16 females $(32 \%)$. Maximum number of patients belonged to age group of $>17-84$ years. Mean age of ESRD patients was $48 \pm 42.8$. Hypertension $(37 \%)$ was leading cause of ESRD, other causes included diabetes $(21 \%)$, diabetes with hypertension (10\%), chronic glomerulonephritis $(8.6 \%)$, polycystic kidney disease $(7.1 \%)$, obstructive uropathy $(5.7 \%)$, analgesic nephropathy (2.9\%) and aetiology remained unknown in $7.1 \%$ cases. The patients were classified into 1 to 5 stages according to the NKF- KDOQI Figure 4.5 shows the percent distribution of the patients in each stage of CKD. The highest number of the patients belonged to stage 5 (49 patients, ) followed by stage 4 ( 1 patients,), stage 3 (Opatients), and then stage 2(0 patients,). Stage1 (0 patient).

Mean haemoglobin percentage was $9.51 \pm 1.15$ $\mathrm{mg} / \mathrm{dl}$. Mean blood urea level was $172.44 \pm 68.6184$ $\mathrm{mg} \%$. Mean serum creatinine level was $10.304 \pm 3.63$ $\mathrm{mg} / \mathrm{dl}$. Mean serum cholesterol level was $145.08 \pm$ $45.3538 \mathrm{mg} / \mathrm{dl}$. Serum cholesterol level was elevated in $78 \%$ of patients. Mean serum triglyceride level was $146.102 \pm 85.6922 \mathrm{mg} / \mathrm{dl}$; it was elevated in $72 \%$ of 
patients. Mean serum HDL level was $36 \pm 34 \mathrm{mg} / \mathrm{dl}$; it was elevated in $77.1 \%$ of patients. Mean serum HDL level was $35.67 \pm 6.11 \mathrm{mg} / \mathrm{dl}$; it was decreased in $67.1 \%$ of patients.

ECG finding showing normal $10(20 \%)$ patients, sinus tachycardia 26(52\%) patients, tall T-wave $10(20 \%)$ patients, and poor R-wave $4(8 \%)$ patients. was noted. Patients were categorised into two groups; first having serum cholesterol more than $200 \mathrm{mg} / \mathrm{dl}$ and second having serum cholesterol less than $200 \mathrm{mg} / \mathrm{dl}$. Correlation echo finding in 50 patent of ckd pt among HD Concentric LVH ( $\mathrm{n}=56 \%$ ), mild MR,TR $(\mathrm{n}=20 \%)$,puller effusion $(\mathrm{n}=16 \%)$ and control group under $(\mathrm{n}=8 \%)$ Following parameters of echocardiography were studied and analysed in detail; these were left ventricular internal diameter in systole and diastole, interventricular septum diameter in systole, left ventricular posterior wall diameter.

Hypertension was considered when blood pressure was more than $140 / 90 \mathrm{mmHg}$. All patients were categorised into hypertensive and normotensive group according to blood pressure. Findings of 2D- echo were compared among hypertensive and normotensive ESRD patients albuminuria, the majority of the patients $86 \%$ (43) had albuminuria and normal14\%(7)patient.

\section{Recommendations:-}

> Patients with CKD should be classified into stages according to the NKF- KDOQI and routinely tested for GFR and ACR levels to observe the progression of the disease.

$>$ Regular evaluation of CVD risk factors in CKD patients is needed for early diagnosis of these risk factors.

$>$ Aggressive management of CVD risk factors, especially modifiable risk factors such as: hypertension, anaemia, dyslipidemia, and physical inactivity in early stages, is important in controlling the disease progression and may reduce the CVD events.

$>$ Increase attention to CVD events, even at early stages of CKD, not only by nephrologists, but more importantly by cardiologists and the general medical community.

$>$ Encourage the general physician for early referral of the patients with primary kidney deterioration to the nephrology centers will enable early prevention of CVD.
Increase awareness of CKD and its complications and methods of control and prevention, on the level of public community, is important in reducing its adverse consequences.

$>$ Further studies are needed to investigate other emerging cardiovascular risk factors, such as homocysteinaemia, High sensitivity C-reactive protein, lipoprotein (a), and oxidative stress. ultrasonographic measurements such as the measurement of increased intima-media thickness (IMT) of large arteries, is important as early marker and sensitive predictor of CVD events in CKD patients.

$>$ Regular reporting and documentation on the incidence and prevalence of $\mathrm{CKD}$, in our community.

\section{BIBLIOGRAPHY}

1. 1.John T.Daugridas peter G.Blake Todd S.Ing Hand book of dialysis chapter $37 \mathrm{p}$ no 626-645.

2. Kidney Disease Outcomes Quality Initiative (K/DOQI). 2004. K/DOQI clinical practice guidelines on hypertension and antihypertensive agents in chronic kidney disease. American Journal of Kidney Disease,.

3. Amaresan M. 2005. Cardiovascular disease in chronic kidney disease. Indian Journal of Nephrology,:

4. American Diabetes Association (ADA). 2004. Nephropathy in Diabetes. Diabetes Care,

5. Anderson KM, Wilson PW, Odell PM. 1991. An updated coronary risk profile: a statement for health professionals. Circulation

6. Annear N, Banerjee D, Joseph J, Harries T, Rahman S. 2008. Prevalence of chronic kidney disease stages 3-5 among acute medical admissions: another opportunity for screening, Q Journal of Medicine,

7. Blankestijn P. 2005. Effects of Eprosartan in Chronic Kidney Disease. Journal of Clinical Basic Cardiology,

8. Bonnie K, Kronenberg F, Beddhu S, Cheung A. 2007. Lipoprotein Metabolism and Lipid Management in Chronic Kidney Disease.

9. Journal of American Society of Nephrology, 18: 1246-1261.Braun J, Oldendorf M, Moshage W, Heidler R, Zeitler E, Luft FC. 1996. 
10. Electron beam computed tomography in the evaluation of cardiac calcification in chronic dialysis patients. American Journal of Kidney Disease,: 394

11. Flack M, Neaton D, Daniels B, Esunge P. 1993. Ethnicity and renal disease: Lessons from the Multiple Risk Factor Intervention

12. Trial and the Treatment of Mild Hypertension Study. American Journal of Kidney Disease,.

13. Food and drug administration (FDA). 2004. Risk Factors for CardiovascularDisease.(www.fda.gov/hearthealth/ riskfactors/riskfactors.html). Accessed on may, 2009.

14. Furth S, Cole S, Mims M, Kaskel F, Mak R, Schwartz G, Wong C, Mun A, Warady B. 2006. Design and Methods of the Chronic Kidney Disease in Children (CKiD) Prospective Cohort Study, Clinical Journal of American Society of Nephrology : 1006-1015.

15. Gerstein HC, Mann JF, Yi Q. 2001. Albuminuria and risk of cardiovascular events, death, and heart failure in diabetic and nondiabetic individuals. Journal of American Medical Association; 286: $421-426$.

16. Agraharkar M. 2007. Acute renal failure. Emedicine. (www.emedicine.com/MED/topic1595.htm). Accessed on June 8, 2009

17. Glassock R. 1987. Clinical aspects of glomerular diseases, American journals of kidney disease, (181).

18. (www.fda.gov/hearthealth/riskfactors/riskfactors.h tml). Accessed on may 22, 2014.

19. Furth S, Cole S, Mims M, Kaskel F, Mak R, Schwartz G, Wong C, Mun A, Warady B. 2006. Design and Methods of the Chronic Kidney Disease in Children (CKiD) Prospective Cohort Study, Clinical Journal of American Society of Nephrology 1: 1006-1015.

20. 20.Goicoechea M, De Vinuesa SG, GomezCampdera F, Luno J. 2005. Predictive cardiovascular risk factors in patients with chronic kidney disease (CKD). Kidney international supplement, 39: 35-38.

21. Gerstein HC, Mann JF, Yi Q. 2001. Albuminuria and risk of cardiovascular events, death, and heart failure in diabetic and nondiabetic individuals. Journal of American Medical Association; 286: 421-426.

22. Glassock R. 1987. Clinical aspects of glomerular diseases, American journals of kidney disease, 10 (181).

23. Gulati S. 2008. Chronic Kidney Disease. Emedicine.

(www.emedicine.com/ped/topic3086.htm). Accessed on June 8, 2009.

24. Kaplan A, Szabo 1. 1983. Clinical chemistry: interpretation and techniques, second edition, Lea and Febiger, chapter 4, p 109-110.

25. Longenecker J,Coresh J, Powe N. 2002. Traditional cardiovascular disease risk factors in dialysis patients compared with the general population: The CHOICE study, American Society of Nephrology 13: 1918-1927.

26. Levin A, Singer J, Thompson CR. 1996. Prevalent left ventricular hypertrophy in the predialysis population: identifying opportunities for intervention. American Journal of Kidney Disease, 27: 347-354.

27. National Kidney Foundation (NKF). 2003. K/DOQI clinical practice guidelines on managing dyslipidemias in chronic kidney disease. American Journal of Kidney Diseases, 41 (3): 177.

28. National Kidney Foundation (NKF). 2005. K/DOQI Clinical Practice Guidelines for Bone Metabolism and Disease With Chronic Kidney Disease. American Journal of Kidney Diseases, 46 (4): 1-121.

29. World Health Organization (WHO). 2000. Obesity: Preventing and managing the global epidemic, WHO obesity technical report, Geneva, Switzerland

\section{Abbreviations}

ACE-I: Angiotensin Converting Enzymeinhibitor

ALP: Alkaline phosphatise

AMI: Acute myocardial infarction

ARBS: Angiotensin receptor blockers

AVC: Aortic valve calcification

BB:Beta blockers

BP: Blood Pressure Ca: Calcium

$\mathrm{CaMB} \quad$ : Ca mass balance

Ca x P: Calcium Phosphorus product

CAD: Coronary Artery disease 
CaR: calcium sensing receptor

CHD: Coronary heart disease

Name:

CHF: Congestive heart failure

Age/ Sex:

CI: Collapse Index

Address:

CKD: Chronic Kidney Disease

ward/ bed/ opd:

CKD-MBD: Chronic kidney disease -mineral Date:

bonedisorder

Presenting complaints:

CRP: C-reactive protein

H/o present illness:

CV: Cardiovascular

Past history of:

CVD: Cardiovascular disease

Any renal disease:

dCa: Dialysate calcium

DCOR: Dialysis Clinical Outcomes Revisited

ECG: Electrocardiography

ESRD: End stage renal disease

FDA: Food and drug association

FGF 23: Fibroblast growth factor

Any infectious disease:

Any chronic medical disease:

Personal history:

Diet:

GFR : Glomerular Filtration Rate

Sleep:

Bowel/bladder habits:

HD: Hemodialysis

HdCa: High calcium dialysate

HDF: Haemodiafiltration

Smoking/ alcoholism:

IDWG: Interdialytic weight gain

IDWG: Interdialytic weight gain

IU: International unit

K : Potassium

Treatment history:

For DM:

For Hypertension:

Family history of:

DM:

HTN:

K/DOQI: Kidney Disease Outcomes Quality Initiative General:

KDIGO: Kidney Disease: Improving Global Weight

Outcomes

LDL Low density lipoproteins

PLHD: Long haemodialysis

LV: Left ventricle

LVH : Left ventricular Hypertrophy

MAC: Mitral annular calcification

Pulse/BP

Cyanosis / Clubbing

Oedema

Systemic Examination

Respiratory

Cardiovascular

Per abdomen

10. PROFORMA

Blood investigation:-

$-\mathrm{Hb}$.

PT. J. N. M. Medical College \& DKS Post

-TLC.

Graduate Institute \&Research Centre\& DR. B.R. -DLC.

A. M. Hospital, Raipur (C.G)

NEPHROLOGY UNIT

DEPARTMENT OF MEDICINE

-RFF.

-Electrolytes.

-Lipid profile.

-Thyroid profile.

"CVS ABNORMALITY IN CKD PATIENT ON HEMODIALYSIS IN TRIBAL POPULATION"

Guide : Dr. Punit Gupta

M.D., D.M., Ph.D., FISN, FISP, MBA

Urine examination:-

-24 hrs. Urine output.

GFR Calculation:-

-MDRD formula.

Physical examination:-

-ECG

-Echo

Diagnosis 